\title{
Semidefinite Programs on Sparse Random Graphs and Their Application to Community Detection
}

\author{
Andrea Montanari \\ Department of Electrical Engineering and \\ Department of Statistics \\ Stanford University \\ USA \\ montanari@stanford.edu
}

\author{
Subhabrata Sen \\ Department of Statistics \\ Stanford University \\ USA \\ ssen90@stanford.edu
}

\begin{abstract}
Denote by $\boldsymbol{A}$ the adjacency matrix of an Erdős-Rényi graph with bounded average degree. We consider the problem of maximizing $\langle\boldsymbol{A}-\mathbb{E}\{\boldsymbol{A}\}, \boldsymbol{X}\rangle$ over the set of positive semidefinite matrices $\boldsymbol{X}$ with diagonal entries $X_{i i}=1$. We prove that for large (bounded) average degree $d$, the value of this semidefinite program (SDP) is - with high probability $-2 n \sqrt{d}+$ $n o(\sqrt{d})+o(n)$. For a random regular graph of degree $d$, we prove that the SDP value is $2 n \sqrt{d-1}+o(n)$, matching a spectral upper bound. Informally, Erdős-Rényi graphs appear to behave similarly to random regular graphs for semidefinite programming.

We next consider the sparse, two-groups, symmetric community detection problem (also known as planted partition). We establish that SDP achieves the information-theoretically optimal detection threshold for large (bounded) degree. Namely, under this model, the vertex set is partitioned into subsets of size $n / 2$, with edge probability $a / n$ (within group) and $b / n$ (across). We prove that SDP detects the partition with high probability provided $(a-b)^{2} /(4 d)>1+o_{d}(1)$, with $d=$ $(a+b) / 2$. By comparison, the information theoretic threshold for detecting the hidden partition is $(a-b)^{2} /(4 d)>1$ : SDP is nearly optimal for large bounded average degree.

Our proof is based on tools from different research areas: (i) A new 'higher-rank' Grothendieck inequality for symmetric matrices; (ii) An interpolation method inspired from statistical physics; (iii) An analysis of the eigenvectors of deformed Gaussian random matrices.
\end{abstract}

\section{Categories and Subject Descriptors}

G.1.6 [Numerical Analysis]: Optimization; G.2.2 [Discrete Mathematics]: Graph TheoryGraph Algorithms

\section{Keywords}

Semidefinite Programming Relaxation, Community Detection, Phase Transitions

Permission to make digital or hard copies of all or part of this work for personal or classroom use is granted without fee provided that copies are not made or distributed for profit or commercial advantage and that copies bear this notice and the full citation on the first page. Copyrights for components of this work owned by others than ACM must be honored. Abstracting with credit is permitted. To copy otherwise, or republish, to post on servers or to redistribute to lists, requires prior specific permission and/or a fee. Request permissions from Permissions@acm.org.

STOC'16, June 19-21, 2016, Cambridge, MA, USA

(c) 2016 ACM. $978-1-4503-4132-5 / 16 / 06 \ldots \$ 15.00$

http://dx.doi.org/10.1145/2897518.2897548

\section{INTRODUCTION AND MAIN RESULTS}

\subsection{Background}

Let $G=(V, E)$ be a random graph with vertex set $V=$ $[n]$, and let $\boldsymbol{A}_{G} \in\{0,1\}^{n \times n}$ denote its adjacency matrix. Spectral algorithms have proven extremely successful in analyzing the structure of such graphs under various probabilistic models. Interesting tasks include finding clusters, communities, latent representations, collaborative filtering and so on $[3,48,54,21]$. The underlying mathematical justification for these applications can be informally summarized as follows (more precise statements are given below):

If $G$ is dense enough, then $\boldsymbol{A}_{G}-\mathbb{E}\left\{\boldsymbol{A}_{G}\right\}$ is much smaller, in operator norm, than $\mathbb{E}\left\{\boldsymbol{A}_{G}\right\}$.

(Recall that the operator norm of a symmetric matrix $\boldsymbol{M}$ is $\|\boldsymbol{M}\|_{o p}=\max \left(\xi_{1}(\boldsymbol{M}),-\xi_{n}(\boldsymbol{M})\right)$, with $\xi_{\ell}(\boldsymbol{M})$ the $\ell$-th largest eigenvalue of $\boldsymbol{M}$.)

Random regular graphs provide the simplest model on which this intuition can be made precise Denoting by $\mathrm{G}^{\text {reg }}(n, d)$ the uniform distribution over graphs with $n$ vertices and uniform degree $d$, we have, for $G \sim \mathrm{G}^{\text {reg }}(n, d), \mathbb{E} \boldsymbol{A}_{G} \approx(d / n) \mathbf{1 1}^{\top}$, whence $\left\|\mathbb{E} \boldsymbol{A}_{G}\right\|_{2} \approx d$. On the other hand, the fact that random regular graphs are 'almost Ramanujan' [29] implies $\left\|\boldsymbol{A}_{G}-\mathbb{E} \boldsymbol{A}_{G}\right\|_{o p} \leq 2 \sqrt{d-1}+o_{n}(1) \ll d$. Roughly speaking, the random part $\boldsymbol{A}_{G}-\mathbb{E} \boldsymbol{A}_{G}$ is smaller than the expectation by a factor $2 / \sqrt{d}$.

The situation is not as clean-cut for random graph with irregular degrees. To be definite, consider the Erdős-Rényi random graph distribution $\mathrm{G}(n, d / n)$ whereby each edge is present independently with probability $d / n$ (and hence the average degree is roughly $d$ ). Also in this case $\mathbb{E} \boldsymbol{A}_{G} \approx$ $(d / n) \mathbf{1 1}{ }^{\top}$, whence $\left\|\mathbb{E} \boldsymbol{A}_{G}\right\|_{o p} \approx d$. However, the largest eigenvalue of $\boldsymbol{A}_{G}-\mathbb{E} A_{G}$ is of the order of the square root of the maximum degree, namely $\sqrt{\log n /(\log \log n)}$ [43]. Summarizing

$\left\|A_{G}-\mathbb{E} A_{G}\right\|_{o p}= \begin{cases}2 \sqrt{d-1}(1+o(1)) & \text { if } G \sim \mathrm{G}^{\mathrm{reg}}(n, d), \\ \sqrt{\frac{\log n}{\log \log n}}(1+o(1)) & \text { if } G \sim \mathrm{G}(n, d / n) .\end{cases}$

Further, for $G \sim \mathrm{G}(n, d / n)$, the leading eigenvectors of $\boldsymbol{A}_{G}-$ $\mathbb{E} \boldsymbol{A}_{G}$ are concentrated near to high-degree vertices, and carry virtually no information about the global structure of $G$. In particular, they cannot be used for clustering.

Far from being a mathematical curiosity, this difference has far-reaching consequences: spectral algorithms are known 
fail, or to be vastly suboptimal for random graphs with bounded average degree $[25,22,40,23,44]$. The community detection problem (a.k.a. 'planted partition') is an example of this failure that attracted significant attention recently. Let $\mathrm{G}(n, a / n, b / n)$ be the distribution over graph with $n$ vertices defined as follows. The vertex set is partitioned uniformly at random into two subsets $S_{1}, S_{2}$ with $\left|S_{i}\right|=n / 2$. Conditional on this partition, edges are independent with

$$
\mathbb{P}\left((i, j) \in E \mid S_{1}, S_{2}\right)= \begin{cases}a / n & \text { if }\{i, j\} \subseteq S_{1} \text { or }\{i, j\} \subseteq S_{2} \\ b / n & \text { otherwise }\end{cases}
$$

Given a single realization of such a graph, we would like to detect, and identify the partition. Early work on this problem showed that simple spectral methods are successful when $a=a(n), b=b(n) \rightarrow \infty$ sufficiently fast. However Eq. (1) -and its analogue for the model $\mathrm{G}(n, a / n, b / n)$ - implies that this approach fails unless $(a-b)^{2} \geq C \log n / \log \log n$. (Throughout $C$ indicates numerical constants.)

Several ideas have been developed to overcome this difficulty. The simplest one is to simply remove from $G$ all vertices whose degree is -say- more than ten times larger than the average degree $d$. Feige and Ofek [25] showed that, if this procedure is applied to $G \sim \mathrm{G}(n, d / n)$, it yields a new graph $G^{\prime}$ that has roughly the same number of vertices as $G$, but $\left\|\boldsymbol{A}_{G}-\mathbb{E}\left\{\boldsymbol{A}_{G}\right\}\right\|_{o p} \leq C \sqrt{d}$, with high probability. The same trimming procedure was successfully applied in [40] to matrix completion, and in $[22,19]$ to community detection. This approach has however several drawbacks. First, the specific threshold for trimming is somewhat arbitrary and relies on the idea that degrees should concentrate around their average: this is not necessarily true in actual applications. Second, it discards a subset of the data. Finally, it is only optimal 'up to constants.'

A new set of spectral methods to overcome the same problem were proposed and analyzed within the community detection problem $[23,44,52,47,13,45]$. These methods construct a new matrix that replaces the adjacency matrix $\boldsymbol{A}_{G}$, and then compute its leading eigenvalues/eigenvectors. We refer to Section 2 for further discussion. These approaches are extremely interesting and mathematically sophisticated. In particular, some of them have been proved to have an optimal detection threshold under the model $\mathrm{G}(n, a / n, b / n)$ $[52,47,13]$. Unfortunately they rely on delicate properties of the underlying probabilistic model. For instance, they are not robust to an adversarial addition of $o(n)$ edges (see Section 4).

\subsection{Main Results (I): Erdôs-Rényi and Ran- dom Regular Graphs}

Semidefinite programming (SDP) relaxations provide a different approach towards overcoming the limitations of spectral algorithms. We denote the cone of $n \times n$ symmetric positive semidefinite matrice by $\operatorname{PSD}(n) \equiv\left\{\boldsymbol{X} \in \mathbb{R}^{n \times n}\right.$ : $\boldsymbol{X} \succeq 0\}$. The convex set of positive-semidefinite matrices with diagonal entries equal to one is denoted by

$$
\mathrm{PSD}_{1}(n) \equiv\left\{\boldsymbol{X} \in \mathbb{R}^{n \times n}: \boldsymbol{X} \succeq 0, X_{i i}=1 \forall i \in[n]\right\}
$$

The set $\operatorname{PSD}_{1}(n)$ is also known as the elliptope. Given a matrix $\boldsymbol{M}$, we define ${ }^{1}$

$$
\operatorname{SDP}(\boldsymbol{M}) \equiv \max \left\{\langle\boldsymbol{M}, \boldsymbol{X}\rangle: \quad \boldsymbol{X} \in \mathrm{PSD}_{1}(n)\right\} .
$$

It is well known that approximate information about the extremal cuts of $G$ can be obtained by computing $\operatorname{SDP}\left(\boldsymbol{A}_{G}\right)$ [30].

The main result of this paper is that the above SDP is also nearly optimal in extracting information about sparse random graphs. In particular, it eliminates the irregularities due to high-degree vertices, cf. Eq. (1). Our first result characterizes the value of $\operatorname{SDP}\left(\boldsymbol{A}_{G}-\mathbb{E}\left\{\boldsymbol{A}_{G}\right\}\right)$ for $G$ an ErdösRényi random graph with large bounded degree ${ }^{2}$. (Its proof is given in Appendix A.)

Theorem 1. Let $G \sim \mathrm{G}(n, d / n)$ be an Erdös-Rényi random graph with edge probability $d / n, \boldsymbol{A}_{G}$ its adjacency matrix, and $\boldsymbol{A}_{G}^{c e n} \equiv \boldsymbol{A}_{G}-\mathbb{E}\left\{\boldsymbol{A}_{G}\right\}$ its centered adjacency matrix. Then there exists $C=C(d)$ such that with probability at least $1-C e^{-n / C}$, we have

$$
\begin{aligned}
\frac{1}{n} \operatorname{SDP}\left(\boldsymbol{A}_{G}^{c e n}\right) & =2 \sqrt{d}+o_{d}(\sqrt{d}), \\
\frac{1}{n} \operatorname{SDP}\left(-\boldsymbol{A}_{G}^{c e n}\right) & =2 \sqrt{d}+o_{d}(\sqrt{d}) .
\end{aligned}
$$

Note that $\operatorname{SDP}\left(\boldsymbol{A}_{G}^{\text {cen }}\right) \leq n \xi_{1}\left(\boldsymbol{A}_{G}^{\text {cen }}\right)$ (here and in the following $\xi_{1}(\boldsymbol{M}) \geq \xi_{2}(\boldsymbol{M}) \geq \ldots \xi_{n}(\boldsymbol{M})$ denote the eigenvalues of the symmetric matrix $\boldsymbol{M})$. However, while $\xi_{1}\left(\boldsymbol{A}_{G}^{\text {cen }}\right)$ is sensitive to vertices of atypically large degree, cf. Eq. (1), $\operatorname{SDP}\left(\boldsymbol{A}_{G}^{\text {cen }}\right)$ appears to be sensitive only to the average degree. Intuitively, the constraint $X_{i i}=1$ rules out the highly localized eigenvectors that are responsible for $\xi_{1}\left(\boldsymbol{A}_{G}^{\text {cen }}\right) \approx$ $\sqrt{\log n / \log \log n}$.

Another way of interpreting Theorem 1 is that ErdösRényi random graphs behave, with respect to SDP as random regular graphs with the same average degree. Indeed, we have the following more precise result for regular graphs. (We omit the proof due to space limitations.)

THEOREM 2. Let $G \sim \mathrm{G}^{\mathrm{reg}}(n, d)$ be a random regular graph with degree d, and $\boldsymbol{A}_{G}^{\text {cen }} \equiv \boldsymbol{A}_{G}-\mathbb{E}\left\{\boldsymbol{A}_{G}\right\}$ its centered adjacency matrix. Then, with high probability

$$
\begin{aligned}
\frac{1}{n} \operatorname{SDP}\left(\boldsymbol{A}_{G}^{c e n}\right) & =2 \sqrt{d-1}+o_{n}(1), \\
\frac{1}{n} \operatorname{SDP}\left(-\boldsymbol{A}_{G}^{c e n}\right) & =2 \sqrt{d-1}+o_{n}(1) .
\end{aligned}
$$

Remark 1. The quantity $\operatorname{SDP}\left(\boldsymbol{A}_{G}^{\text {cen }}\right)$ can also be thought as a relaxation of the problem of maximizing $\sum_{i, j=1}^{n} A_{i j} \sigma_{i} \sigma_{j}$ over $\sigma_{i} \in\{+1,-1\}, \sum_{i=1}^{n} \sigma_{i}=0$. The result of our companion paper [24] implies that this has - with high probabilityvalue $2 n \mathrm{P}_{*} \sqrt{d}+n o_{d}(\sqrt{d})$ (see [24] for a definition of $\mathrm{P}_{*}$ ). We deduce that - with high probability - the SDP relaxation overestimates the optimum by a factor $1 / \mathrm{P}_{*}+o_{d}(1)$ (where $\left.1 / \mathrm{P}_{*} \approx 1.310\right)$.

\footnotetext{
${ }^{1}$ Here and below $\langle\boldsymbol{A}, \boldsymbol{B}\rangle=\operatorname{Tr}\left(\boldsymbol{A}^{\top} \boldsymbol{B}\right)$ is the usual scalar product between matrices.

${ }^{2}$ Throughout the paper, $O(\cdot), o(\cdot)$, and $\Theta(\cdot)$ refer to the usual $n \rightarrow \infty$ asymptotic, while $O_{d}(\cdot), o_{d}(\cdot)$ and $\Theta_{d}(\cdot)$ are used to describe the $d \rightarrow \infty$ asymptotic regime. We say that a sequence of events $B_{n}$ occurs with high probability (w.h.p.) if $\mathbb{P}\left(B_{n}\right) \rightarrow 1$ as $n \rightarrow \infty$. Finally, for random $\left\{X_{n}\right\}$ and non-random $f: \mathbb{R}_{>0} \rightarrow \mathbb{R}_{>0}$, we say that $X_{n}=o_{d}(f(d))$ w.h.p. as $n \rightarrow \infty$ if there exists non-random $g(d)=o_{d}(f(d))$ such that the sequence $B_{n}=\left\{\left|X_{n}\right| \leq g(d)\right\}$ occurs w.h.p. $($ as $n \rightarrow \infty)$.
} 
Remark 2. For the sake of simplicity, we stated Eqs. (5), (6), in asymptotic form. However, our proof provides quantitative bounds on the error terms. In particular, the $o_{d}(\sqrt{d})$ term is upper bounded by $C d^{2 / 5} \log (d)$, for $C$ a numerical constant.

\subsection{Main Results (II): Hidden Partition Prob- lem}

We next apply the SDP defined in Eq. (4) to the community detection problem. To be definite we will formalize this as a binary hypothesis testing problem, whereby we want to determine - with high probability of success- whether the random graph under consideration has a community structure or not. The estimation version of the problem, i.e. the question of determining -approximately- a partition into communities, is addressed in Section 4.1 that also proposes a specific algorithm to round the SDP solution, i.e. to construct a partitions of the vertices from the SDP solution.

Within the hypothesis testing formulation, we are given a single graph $G=(V, E)$ over $n$ vertices and we have to decide which of the following holds:

Hypothesis 0: $G \sim \mathrm{G}(n, d / n)$ is an Erdős-Rényi random graph with edge probability $d / n, d=(a+b) / 2$. We denote the corresponding distribution over graphs by $\mathbb{P}_{0}$.

Hypothesis 1: $G \sim \mathrm{G}(n, a / n, b / n)$ is an random graph with a planted partition and edge probabilities $a / n, b / n$. We denote the corresponding distribution over graphs by $\mathbb{P}_{1}$.

A statistical test takes as input a graph $G$, and returns $T(G) \in\{0,1\}$ depending on which hypothesis is estimated to hold. We say that it is successful with high probability if $\mathbb{P}_{0}(T(G)=1)+\mathbb{P}_{1}(T(G)=0) \rightarrow 0$ as $n \rightarrow \infty$.

Theorem 1 indicates that, under Hypothesis 0 , we have $\operatorname{SDP}\left(\boldsymbol{A}_{G}-(d / n) \mathbf{1 1}^{\top}\right)=2 n \sqrt{d}+n o_{d}(\sqrt{d})$. This suggests the following test:

$$
T(G ; \delta)= \begin{cases}1 & \text { if } \operatorname{SDP}\left(\boldsymbol{A}_{G}-(d / n) \mathbf{1 1}^{\top}\right) \geq 2 n(1+\delta) \sqrt{d} \\ 0 & \text { otherwise }\end{cases}
$$

Mossel, Neeman, Sly [51] proved that no test can be successful with high probability if $(a-b)<\sqrt{2(a+b)}$. Polynomially computable tests that achieve this threshold were developed in $[52,47,13]$ using advanced spectral methods. As mentioned, these approaches can be fragile to perturbations of the precise probabilistic model, cf. Section 4.

Our next result addresses the fundamental question: Does the SDP-based test achieve the information theoretic threshold? Recent work of Guédon and Vershynin [33] studies the same question, but requires $(a-b)^{2} \geq C(a+b)$ with $C$ a large constant.

Theorem 3. Assume, for some $\varepsilon>0$,

$$
\frac{a-b}{\sqrt{2(a+b)}} \geq 1+\varepsilon
$$

Then there exists $\delta_{*}=\delta_{*}(\varepsilon)>0$ and $d_{*}=d_{*}(\varepsilon)>0$ such that the following holds. If $d=(a+b) / 2 \geq d_{*}$, then the SDP-based test $T\left(\cdot ; \delta_{*}\right)$ succeeds with high probability.

Further, the error probability is at most $C e^{-n / C}$ for $C=$ $C(a, b)$ a constant.
We refer to Appendix A for the proof of this theorem.

Remark 3. This theorem guarantees that SDP is nearly optimal for large but bounded degree $d$. By comparison, the naive spectral test that returns $T_{\text {spec }}(G)=1$ if $\xi_{1}\left(\boldsymbol{A}_{G}^{\text {cen }}\right) \geq \theta_{*}$ and $T_{\text {spec }}(G)=0$ otherwise (for any threshold value $\theta_{*}$ ) is sub-optimal by an unbounded factor for $d=O(1)$.

Remark 4. One might wonder why we consider large degree asymptotics $d=(a+b) / 2 \rightarrow \infty$ instead of trying to establish a threshold at $(a-b) / \sqrt{2(a+b)}=1$ for fixed $a$, $b$. Preliminary non-rigorous calculation [39], as well as an argument in [50], suggest that indeed this is necessary. For fixed $(a+b)$ the SDP threshold does not coincide with the optimal one.

Remark 5. For the sake of simplicity, we formulated the community detection problem as an hypothesis testing problem. A related (somewhat more challenging) task is to estimate the hidden partition better than by random guessing. In Section 4.1 we will show that, under the same conditions of Theorem 3, we can assign vertices making at most $(1-\Delta) n / 2$ mistakes (with high probability for some $\Delta$ bounded away from 0$)$.

We will discuss related work in the next section, then provide an outline of the proof ideas in Section 3, and finally discuss extension of the above results in Section 4. Detailed proofs are deferred to the appendix.

\subsection{Notations}

Given $n \in \mathbb{N}$, we let $[n]=\{1,2, \ldots, n\}$ denote the set of first $n$ integers. We write $|S|$ for the cardinality of a set $S$. We will use lowercase boldface (e.g. $\boldsymbol{v}=\left(v_{1}, \ldots, v_{n}\right), \boldsymbol{x}=$ $\left(x_{1}, \ldots, x_{n}\right)$, etc.) for vectors and uppercase boldface (e.g. $\boldsymbol{A}=\left(A_{i, j}\right)_{i, j \in[n]}, \boldsymbol{Y}=\left(Y_{i, j}\right)_{i, j \in[n]}$, etc. $)$ for matrices. Given a symmetric matrix $\boldsymbol{M}$, we let $\xi_{1}(\boldsymbol{M}) \geq \xi_{2}(\boldsymbol{M}) \geq \cdots \geq$ $\xi_{n}(\boldsymbol{M})$ be its ordered eigenvalues (with $\xi_{\max }(\boldsymbol{M})=\xi_{1}(\boldsymbol{M})$, $\left.\xi_{\min }(\boldsymbol{M})=\xi_{n}(\boldsymbol{M})\right)$. In particular $\mathbf{1}_{n}=(1,1, \ldots, 1) \in \mathbb{R}^{n}$ is the all-ones vector, $\mathrm{I}_{n}$ the identity matrix, and $\boldsymbol{e}_{i} \in \mathbb{R}^{n}$ is the $i$ 'th standard unit vector.

For $\boldsymbol{v} \in \mathbb{R}^{m},\|\boldsymbol{v}\|_{p}=\left(\sum_{i=1}^{p}\left|v_{i}\right|^{p}\right)^{1 / p}$ denotes its $\ell_{p}$ norm (extendend in the standard way to $p=\infty$ ). For a matrix $\boldsymbol{M}$, we denote by $\|\boldsymbol{M}\|_{p \rightarrow q}=\sup _{\boldsymbol{v} \neq 0}\|\boldsymbol{M v}\|_{q} /\|\boldsymbol{v}\|_{q}$ its $\ell_{p}$-to$\ell_{q}$ operator norm, with the standard shorthands $\|M\|_{o p} \equiv$ $\|M\|_{2} \equiv\|M\|_{2 \rightarrow 2}$.

Throughout with high probability means 'with probability converging to one as $n \rightarrow \infty$.' We follow the standard Big-Oh notation for asymptotics. We will be interested in bounding error terms with respect to $n$ and $d$. Whenever not clear from the contest, we indicate in subscript the variable that is large. For instance $f(n, d)=o_{d}(1)$ means that there exists a function $g(d) \geq 0$ independent of $n$ such that $\lim _{d \rightarrow \infty} g(d)=0$ and $|f(n, d)| \leq g(d)$. (Hence $f(n, d)=$ $\cos (0.1 n) / d=o_{d}(1)$ but $\left.f(n, d)=\log (n) / d \neq o_{d}(1).\right)$

A random graph has a law (distribution), which is a probability distribution over graphs with the same vertex set $V=[n]$. Since we are interested in the $n \rightarrow \infty$ asymptotics, it will be implicitly understood that one such distribution is specified for each $n$.

We will use $C$ (or $C_{0}, C_{1}, \ldots$ ) to denote constants, that will change from point to point. Unless otherwise stated, these are universal constants. 


\section{FURTHER RELATED LITERATURE}

Few results have been proved about the behavior of classical SDP relaxations on sparse random graphs and -to the best of our knowledge- none of these earlier results is tight.

Significant amount of work has been devoted to analyzing SDP hierarchies on random CSP instances [31, 57], and -more recently- on (semi-)random Unique games instances [42]. These papers typically prove only one-side bounds that are not claimed to be sharp as the number of variables diverge.

Coja-Oghlan [20] studies the value of Lovász theta function $\vartheta(G)$, for $G \sim \mathrm{G}(n, p)$ a dense Erdős-Rényi random graph, estabilishing $C_{1} \sqrt{n / p} \leq \vartheta(G) \leq C_{2} \sqrt{n / p}$ with high probability. As in the previous cases, this result is not tight.

Ambainis et al. [5] study an SDP similar to (4), for $\boldsymbol{M}$ a dense random matrix with i.i.d. entries. One of their main results is analogous to a special case of our Theorem 5.(b) below -namely, to the case $\lambda=0$. (We prefer to give an independent -simpler- proof also of this case.)

Several papers have been devoted to SDP approaches for community detection and the related 'synchronization' problem. A partial list includes $[9,1,35,36,7]$. These papers focus on finding sufficient conditions under which the SDP recovers exactly the unknown signal. For instance, in the context of the hidden partition model (2), this requires diverging average degrees $a, b=\Theta(\log n)[1,35,36]$. SDP was proved in [35] to achieve the information-theoretically optimal threshold for exact reconstruction. The techniques to prove this type of result are very different from the ones employed here: since the (conjectured) optimum is known explicitly, it is sufficient to certify it through a dual witness.

The only result on community detection that compares to ours was recently proven in a remarkable paper by Guédon and Vershynin [33]. Their work uses the classical Grothendieck inequality to establish upper bounds on the estimation error of SDP. However, the resulting bound applies only under the condition $(a-b)^{2} \geq 10^{4}(a+b)$. This condition is suboptimal with respect to the information-theoretic threshold $(a-b)^{2}>2(a+b)$ established in [51, 52, 47]. In particular, the results of [33] leave open the central question of practical importance: is SDP to be discarded in favor of the spectral methods of $[52,47]$, or is the sub-optimality just an outcome of the analysis?

In this paper we provide evidence indicating that SDP is in fact nearly optimal for community detection. While we also make use of a Grothendieck inequality as in [33], this is only one step (and not the most challenging) in a significantly longer argument. Indeed, the gap between the ideal threshold at $(a-b) / \sqrt{2(a+b)}=1$, and the guarantees of [33] cannot be filled simply by carrying out more carefully the same proof strategy. In order fill the gap we need to develop several new ideas: ( $i$ ) A new (higher rank) Grothendieck inequality; (ii) A smoothing of the original graph parameter SDP $(\cdot)$; (iii) An interpolation argument; (iv) A sharp analysis of SDP for Gaussian random matrices.

\section{PROOF STRATEGY}

Throughout, we denote by $\boldsymbol{A}_{G}^{\text {cen }}=\boldsymbol{A}_{G}-(d / n) \mathbf{1 1}{ }^{\top}$ the centered adjacency matrix of $G \sim \mathrm{G}(n, d / n)$ or $G \sim \mathrm{G}(n, a / n, b / n)$. Our proofs of Theorem 1 and Theorem 3 follows a similar strategy that can be summarized as follows:

Step 1: Smooth. We replace the function $\boldsymbol{M} \mapsto \operatorname{SDP}(\boldsymbol{M})$, by a smooth function $\boldsymbol{M} \mapsto \Phi(\beta, k ; \boldsymbol{M})$ that depends on two additional parameters $\beta \in \mathbb{R}_{\geq 0}$ and $k \in \mathbb{N}$. We prove that, for $\beta, k$ large (and $\boldsymbol{M}$ sufficiently 'regular'), $|\operatorname{SDP}(\boldsymbol{M})-\Phi(\beta, k ; \boldsymbol{M})|$ can be made arbitrarily small, uniformly in the matrix dimensions. This in particular requires developing a new (higher rank) Grothendiecktype inequality, which is of independent interest, see Section 3.1.

Step 2: Interpolate. We use an interpolation method (analogous to the Lindeberg method) to compare the value $\Phi\left(\beta, k ; \boldsymbol{A}_{G}^{\text {cen }}\right)$ to $\Phi(\beta, k ; \boldsymbol{B})$, where $\boldsymbol{B} \in \mathbb{R}^{n \times n}$ is a symmetric Gaussian matrix with independent entries. More precisely, we use $B_{i j} \sim \mathrm{N}(0,1 / n)$ to approximate $G \sim$ $\mathrm{G}(n, d / n)$ and $B_{i j} \sim \mathrm{N}(\lambda / n, 1 / n)$ to approximate the hidden partition model $G \sim \mathrm{G}(n, a / n, b / n)$, with $\lambda \equiv$ $(a-b) / \sqrt{2(a+b)}$. Further detail is provided in Section 3.2 .

Note that the interpolation/Lindeberg method requires $\boldsymbol{M} \mapsto \Phi(\beta, k ; \boldsymbol{M})$ to be differentiable, which is the reason for Step 1 above.

Step 3: Analyze. We finally carry out an analysis of $\operatorname{SDP}(\boldsymbol{B})$ with $\boldsymbol{B}$ distributed according to the above Gaussian models. In doing this we can take advantage of the high degree of symmetry of Gaussian random matrices. This part of the proof is relatively simple for Theorem 1, but becomes challenging in the case of Theorem 3, see Section 3.3.

(The proof of Theorem 2 is more direct.) In the next subsections we will provide further details about each of these steps. The formal proofs of Theorem 1 and Theorem 3 are presented in Appendix A, with technical lemmas in other appendices..

The construction of the smooth function $\Phi(\beta, k ; \boldsymbol{M})$ is inspired from statistical mechanics. As an intermediate step, define the following rank-constrained version of the SDP (4)

$$
\begin{aligned}
\mathrm{OPT}_{k}(\boldsymbol{M}) & \equiv \max \left\{\langle\boldsymbol{M}, \boldsymbol{X}\rangle: \boldsymbol{X} \in \mathrm{PSD}_{1}(n), \operatorname{rank}(\boldsymbol{X}) \leq k\right\} \\
& =\max \left\{\sum_{i, j=1}^{n} M_{i j}\left\langle\boldsymbol{\sigma}_{i}, \boldsymbol{\sigma}_{j}\right\rangle: \boldsymbol{\sigma}_{i} \in \mathbb{S}^{k-1}\right\}, \quad(11)
\end{aligned}
$$

where $\mathbb{S}^{k-1}=\left\{\boldsymbol{\sigma} \in \mathbb{R}^{k}:\|\boldsymbol{\sigma}\|_{2}=1\right\}$ be the unit sphere in $k$ dimensions. We then define $\Phi(\beta, k ; \boldsymbol{M})$ as the following log-partition function

$\Phi(\beta, k ; \boldsymbol{M}) \equiv \frac{1}{\beta} \log \left\{\int \exp \left\{\beta \sum_{i, j=1}^{n} M_{i j}\left\langle\boldsymbol{\sigma}_{i}, \boldsymbol{\sigma}_{j}\right\rangle\right\} \mathrm{d} \nu(\boldsymbol{\sigma})\right\}$.

Here $\boldsymbol{\sigma}=\left(\boldsymbol{\sigma}_{1}, \boldsymbol{\sigma}_{2}, \ldots, \boldsymbol{\sigma}_{n}\right) \in\left(\mathbb{S}^{k-1}\right)^{n}$ and we denote by $\mathrm{d} \nu(\cdot)$ the uniform measure on $\left(\mathbb{S}^{k-1}\right)^{n}$ (normalized to 1, i.e. $\left.\int \mathrm{d} \nu(\boldsymbol{\sigma})=1\right)$.

It is easy to see that $\lim _{\beta \rightarrow \infty} \Phi(\beta, k ; \boldsymbol{M})=\mathrm{OPT}_{k}(\boldsymbol{M})$, and $\mathrm{OPT}_{n}(\boldsymbol{M})=\operatorname{SDP}(\boldsymbol{M})$. For carrying out the above proof strategy we need to bound the errors $\mid \Phi(\beta, k ; \boldsymbol{M})-$ $\mathrm{OPT}_{k}(\boldsymbol{M}) \mid$ and $\left|\mathrm{OPT}_{k}(\boldsymbol{M})-\operatorname{SDP}(\boldsymbol{M})\right|$ uniformly in $n$.

\subsection{Higher-Rank Grothendieck Inequalities and Zero-Temperature Limit}

In order to bound the error $\left|\mathrm{OPT}_{k}(\boldsymbol{M})-\operatorname{SDP}(\boldsymbol{M})\right|$ we develop a new Grothendieck-type inequality which is of independent interest. 
ThEOREM 4. For $k \geq 1$, let $\boldsymbol{g} \sim \mathrm{N}\left(0, \mathrm{I}_{k} / k\right)$ be a vector with i.i.d. centered normal entries with variance $1 / k$, and define $\alpha_{k} \equiv\left(\mathbb{E}\|\boldsymbol{g}\|_{2}\right)^{2}$.

Then, for any symmetric matrix $M \in \mathbb{R}^{n \times n}$, we have the inequalities

$$
\begin{array}{r}
\operatorname{SDP}(\boldsymbol{M}) \geq \mathrm{OPT}_{k}(\boldsymbol{M}) \geq \alpha_{k} \operatorname{SDP}(\boldsymbol{M})-\left(1-\alpha_{k}\right) \operatorname{SDP}(-\boldsymbol{M}), \\
\mathrm{OPT}_{k}(\boldsymbol{M}) \geq\left(2-\alpha_{k}^{-1}\right) \operatorname{SDP}(\boldsymbol{M})-\left(\alpha_{k}^{-1}-1\right) \mathrm{OPT}_{k}(-\boldsymbol{M}) .
\end{array}
$$

Remark 6. The upper bound in Eq. (13) is trivial. Further, it follows from Cauchy-Schwartz that $\alpha_{k} \in(0,1)$ for all $k$. Also $\|\boldsymbol{g}\|_{2}^{2}$ is a chi-squared random variable with $k$ degrees of freedom and hence

$$
\alpha_{k}=\frac{2 \Gamma((k+1) / 2)^{2}}{k \Gamma(k / 2)^{2}}=1-\frac{1}{2 k}+O\left(1 / k^{2}\right) .
$$

Substituting in Eq. (13) we get, for all $k \geq k_{0}$ with $k_{0}$ a sufficiently large constant, and assuming $\operatorname{SDP}(\boldsymbol{M})>0$,

$$
\left(1-\frac{1}{k}\right) \operatorname{SDP}(\boldsymbol{M})-\frac{1}{k}|\operatorname{SDP}(-\boldsymbol{M})| \leq \mathrm{OPT}_{k}(\boldsymbol{M}) \leq \operatorname{SDP}(\boldsymbol{M}) .
$$

In particular, if $|\operatorname{SDP}(-\boldsymbol{M})|$ is of the same order as $\operatorname{SDP}(\boldsymbol{M})$, we conclude that $\mathrm{OPT}_{k}(\boldsymbol{M})$ approximates $\operatorname{SDP}(\boldsymbol{M})$ with a relative error of order $O(1 / k)$.

The classical Grothendieck inequality concerns non-symmetric bilinear forms [32]. A Grothendieck inequality for symmetric matrices was established in [53, 49] (see also [4] for generalizations) and states that, for a constant $C$,

$$
\mathrm{OPT}_{1}(\boldsymbol{M}) \geq \frac{1}{C \log n} \operatorname{SDP}(\boldsymbol{M}) .
$$

Higher-rank Grothendieck inequalities were developed in the setting of general graphs in $[16,15]$. However, constantfactor approximations were not established for the present problem (which corresponds to the the complete graph case in $[16])$.

Constant factor approximations exist for $\boldsymbol{M}$ positive semidefinite [15]. We note that Theorem 4 implies the inequality of [15]. Using $\operatorname{SDP}(-\boldsymbol{M}) \leq-\xi_{\min }(\boldsymbol{M})$ in Eq. (13), we obtain the inequality of [15] for the positive semidefinite matrix $\boldsymbol{M}-\xi_{\min }(\boldsymbol{M}) \mathrm{I}$. On the other hand, the result of [15] is too weak for our applications. We want to apply Theorem 4 -among others- to $\boldsymbol{M}=\boldsymbol{A}_{G}^{\text {cen }}$ with $\boldsymbol{A}_{G}^{\text {cen }}$ the adjacency matrix of $G \sim \mathrm{G}(n, d / n)$. This matrix is non-positive definite, and in a dramatic way with smallest eigenvalue satisfying $\left.-\xi_{\min }\left(\boldsymbol{A}_{G}^{\mathrm{cen}}\right) \approx(\log n /(\log \log n))^{1 / 2} \gg \operatorname{SDP}\left(-\boldsymbol{A}_{G}^{\mathrm{cen}}\right)\right)$.

In summary, we could not use the vast literature on Grothen type inequalities to prove our main result, Theorem 1, which motivated us to develop Theorem 4.

Theorem 4 will allow to bound $\left|\operatorname{SDP}(\boldsymbol{M})-\mathrm{OPT}_{k}(\boldsymbol{M})\right|$ for $\boldsymbol{M}$ either a centered adjacency matrix or a Gaussian matrix. The next lemma bounds the 'smoothing error' $\mid \Phi(\beta, k ; \boldsymbol{M})-$ $\mathrm{OPT}_{k}(\boldsymbol{M}) \mid$.

LEMMA 3.1. There exists an absolute constant $C$ such that for any $\varepsilon \in(0,1]$ the following holds. If $\|\boldsymbol{M}\|_{\infty \rightarrow 2} \equiv$ $\max \left\{\|\boldsymbol{M x}\|_{2}: \quad\|\boldsymbol{x}\|_{\infty} \leq 1\right\} \leq L \sqrt{n}$, then

$$
\left|\frac{1}{n} \Phi(\beta, k ; M)-\frac{1}{n} \mathrm{OPT}_{k}(\boldsymbol{M})\right| \leq 2 L \varepsilon \sqrt{k}+\frac{k}{\beta} \log \frac{C}{\varepsilon} .
$$

\subsection{Interpolation}

Our next step consists in comparing the adjacency matrix of random graph $G$ with a suitable Gaussian random matrix, and bound the error in the corresponding log-partition function $\Phi(\beta, k ; \cdot)$.

Let us recall the definition of Gaussian orthogonal ensemble $\operatorname{GOE}(n)$. We have $\boldsymbol{W} \sim \operatorname{GOE}(n)$ if $\boldsymbol{W} \in \mathbb{R}^{n \times n}$ is symmetric with $\left\{W_{i, j}\right\}_{1 \leq i \leq j \leq n}$ independent, with distribution $W_{i i} \sim \mathrm{N}(0,2 / n)$ and $W_{i j} \sim \mathrm{N}(0,1 / n)$ for $i<j$. We then define, for $\lambda \geq 0$, the following deformed GOE matrix:

$$
\boldsymbol{B}(\lambda) \equiv \frac{\lambda}{n} \mathbf{1 1 ^ { \top }}+\boldsymbol{W},
$$

where $\boldsymbol{W} \sim \operatorname{GOE}(n)$. The argument $\lambda$ will be omitted if clear from the context. The next lemma establishes the necessary comparison bound. Note that we state it for $G \sim \mathrm{G}(n, a / b, b / n)$ a random graph from the hidden partition model, but it obviously applies to standard Erdős-Rényi random graphs by setting $a=b=d$.

Lemma 3.2. Let $\boldsymbol{A}_{G}^{c e n}=\boldsymbol{A}_{G}-(d / n) \mathbf{1 1}^{\top}$ be the centered adjacency matrix of $G \sim \mathrm{G}(n, a / n, b / n)$, whereby $d=(a+$ b) $/ 2$. Define $\lambda=(a-b) / 2 \sqrt{d}$. Then there exists an absolute constant $n_{0}$ such that, if $n \geq \max \left(n_{0},(15 d)^{2}\right)$,

$\mid \frac{1}{n} \mathbb{E} \Phi\left(\beta, k ; \boldsymbol{A}_{G}^{c e n} / \sqrt{d}\right)-\frac{1}{n} \mathbb{E} \Phi\left((\beta, k ; \boldsymbol{B}(\lambda)) \mid \leq \frac{2 \beta^{2}}{\sqrt{d}}+\frac{8 \lambda^{1 / 2}}{d^{1 / 4}}\right.$.

Note that this lemma bounds the difference in expectation. We will use concentration of measure to transfer this result to a bound holding with high probability.

Interpolation (or 'smart path') methods have a long history in probability theory, dating back to Lindeberg's beautiful proof of the central limit theorem [46]. Since our smoothing construction yields a log-partition function $\Phi(\beta, k ; \boldsymbol{M})$, our calculations are similar to certain proofs in statistical mechanics. A short list of statistical-mechanics inspired results in probabilistic combinatorics includes [27, 28, 10, 55, 34]. In our companion paper [24], we used a similar approach to characterize the limit value of the minimum bisection of Erdős-Rényi and random regular graphs.

\subsection{SDPs for Gaussian Random Matrices}

The last part of our proof analyzes the Gaussian model (19). This type of random matrices have attracted a significant amount of work within statistics (under the name of 'spiked model') and probability theory (as 'deformed Wigner -or GOE- matrices'), aimed at characterizing their eigenvalues and eigenvectors. A very incomplete list of references includes [8, 26, 17, 11, 12, 56, 41]. A key phenomenon unveiled by these works is the so-called Baik-Ben Arous-Peché (or ckBBAP) phase transition. In its simplest form (and applied to the matrix of Eq. (19)) this predicts a phase transition in the largest eigenvalue of $\boldsymbol{B}(\lambda)$

$$
\lim _{n \rightarrow \infty} \xi_{1}(\boldsymbol{B}(\lambda))= \begin{cases}2 & \text { if } \lambda \leq 1 \\ \lambda+\lambda^{-1} & \text { if } \lambda>1\end{cases}
$$

(This limit can be interpreted as holding in probability.) Here, we establish an analogue of this result for the SDP value.

Theorem 5 (Phase transition for GOE matrices). Let $\boldsymbol{B}=\boldsymbol{B}(\lambda) \in \mathbb{R}^{n \times n}$ be a symmetric matrix distributed according to the model (19). Namely $\boldsymbol{B}=\boldsymbol{B}^{\top}$ with $\left\{B_{i j}\right\}_{i \leq j}$ 
independent random variables, where $B_{i j} \sim \mathrm{N}(\lambda / n, 1 / n)$ for $1 \leq i<j \leq n$ and $B_{i i} \sim \mathrm{N}(\lambda / n, 2 / n)$ for $1 \leq i \leq n$. Then

(a) If $\lambda \in[0,1]$, then for any $\varepsilon>0$, we have

$$
\frac{1}{n} \operatorname{SDP}(\boldsymbol{B}(\lambda)) \in[2-\varepsilon, 2+\varepsilon]
$$

with probability converging to one as $n \rightarrow \infty$.

(b) If $\lambda>1$, then there exists $\Delta(\lambda)>0$ such that

$$
\frac{1}{n} \operatorname{SDP}(\boldsymbol{B}(\lambda)) / n \geq 2+\Delta(\lambda)
$$

with probability converging to one as $n \rightarrow \infty$.

As mentioned above, we obviously have $\operatorname{SDP}(\boldsymbol{B}) / n \leq$ $\xi_{1}(\boldsymbol{B})$. The first part of this theorem (in conjunction with Eq. (21)) establishes that the upper bound is essentially tight of $\lambda \leq 1$. On the other hand, we expect the eigenvalue upper bound not to be tight for $\lambda>1$ [39]. Nevertheless, the second part of our theorem establishes a phase transition taking place at $\lambda=1$ as for the leading eigenvalue.

Remark 7. The phase transition in the leading eigenvalue has a high degree of universality. In particular, Eq. (21) remains correct if the model (19) is replaced by $\boldsymbol{B}^{\prime}=\lambda \boldsymbol{v} \boldsymbol{v}^{\top}+$ $\boldsymbol{W}$, with $\boldsymbol{v}$ an arbitrary unit vector. On the other hand, we expect the phase transition in $\operatorname{SDP}\left(\boldsymbol{B}^{\prime}\right) / n$ to depend -in general- on the vector $\boldsymbol{v}$, and in particular on how 'spiky' this is.

\section{OTHER RESULTS AND GENERALIZA- TIONS}

While our was focused on a relatively simple model, the techniques presented here allow for several generalizations. We discuss them briefly here.

\subsection{Estimation}

For the sake of simplicity, we formulated community detection as a hypothesis testing problem. It is interesting to consider the associated estimation problem, that requires to estimate the hidden partition $V=S_{1} \cup S_{2}$.

We encode the ground truth using the vector $\boldsymbol{x}_{0} \in\{+1,-1\}^{n}$, with $x_{0, i}=+1$ if $i \in S_{1}$, and $x_{0, i}=-1$ if $i \in S_{2}$. An estimator is a $\operatorname{map}^{3} \widehat{\boldsymbol{x}}: \mathcal{G}_{n} \rightarrow\{+1,0,-1\}^{n}$ with $\mathcal{G}_{n}$ the space of graphs over $n$ vertices. It is proved in [51] that no estimator is substantially better than random guessing for $G \sim \mathrm{G}(n, a / n, b / n)$, with $\lambda=(a-b) / \sqrt{2(a+b)}<1$. More precisely, for $\lambda<1$, any estimator achieves vanishing correlation with the ground truth: $\left|\left\langle\widehat{\boldsymbol{x}}(G), \boldsymbol{x}_{0}\right\rangle\right|=o(n)$ with high probability.

We construct a randomized SDP-based estimator $\hat{\boldsymbol{x}}^{\mathrm{SDP}}(G)$ as follows (we will denote expectation and probability with respect to tha algorithm's randomness by $\mathbb{E}_{\text {alg }}(\cdot)$ and $\left.\mathbb{P}_{\text {alg }}(\cdot)\right)$ :

(i) Partition the edge set $E=E_{1} \cup E_{2}$ by letting $(i, j) \in$ $E_{2}$ independently for each edge $(i, j) \in E$, with probability $\mathbb{P}_{\text {alg }}\left((i, j) \in E_{2}\right)=\delta_{n} /\left(1+\delta_{n}\right), \delta_{n}=n^{-1 / 2}$, and $(i, j) \in E_{1}$ otherwise. Denote by $G_{1}=\left(V, E_{1}\right)$, and $G_{2}=\left(V, E_{2}\right)$ the resulting graphs.

\footnotetext{
${ }^{3}$ Earlier work sometimes assumes $\widehat{\boldsymbol{x}}: \mathcal{G}_{n} \rightarrow\{+1,-1\}^{n}$, i.e. forbids the estimate 0. For our purposes, the two formulations are equivalent: we can always 'simulate' $\hat{x}_{i}=0$ by letting $\hat{x}_{i} \in\{+1,-1\}$ uniformly at random.
}

(ii) Compute an optimizer $\boldsymbol{X}_{*}$ of the SDP (4), $\boldsymbol{M}=\boldsymbol{A}_{G_{1}}^{\text {cen }}$ (i.e. a matrix $\boldsymbol{X}_{*} \in \mathrm{PSD}_{1}(n)$ such that $\left\langle\boldsymbol{A}_{G_{1}}^{\text {cen }}, \boldsymbol{X}_{*}\right\rangle=$ $\left.\operatorname{SDP}\left(\boldsymbol{A}_{G_{1}}^{\text {cen }}\right)\right)$.

(iii) Compute the eigenvalue decomposition $\boldsymbol{X}_{*}=\sum_{i=1}^{n} \xi_{i} \boldsymbol{v}_{i} \boldsymbol{v}_{i}^{\top}$, and let $\boldsymbol{v}_{i}=\left(v_{i, 1}, v_{i, 2}, \ldots, v_{i, n}\right)$ denote the $i$-th eigenvector. For each $i, j \in[n]$ define $\widehat{\boldsymbol{x}}^{(i, j)} \in\{+1,0,-1\}$ by $\widehat{x}_{\ell}^{(i, j)}=\operatorname{sign}\left(\boldsymbol{v}_{i}\right)_{\ell}$ if $\left|v_{i, \ell}\right| \geq\left|v_{i, j}\right|$ and $\widehat{x}_{\ell}^{(i, j)}=0$ otherwise. (In words, $\widehat{\boldsymbol{x}}^{(i, j)}$ is obtained from $\boldsymbol{v}_{i}$ by zeroing entries with magnituude below $\left|v_{i, j}\right|$ and taking the sign of those above).

(iv) Select $(I, J)=\arg \max _{i, j \in[n]}\left\langle\widehat{\boldsymbol{x}}^{(i, j)}, \boldsymbol{A}_{G_{2}}^{\mathrm{cen}} \widehat{\boldsymbol{x}}^{(i, j)}\right\rangle$, and return $\hat{\boldsymbol{x}}^{\mathrm{SDP}}(G)=\widehat{\boldsymbol{x}}^{(I, J)}$.

The next results implies that -for large bounded average degree $d$ - this estimator has a nearly optimal threshold.

THEOREM 6. Let $G \sim \mathrm{G}(n, a / n, b / n)$ and assume, for some $\varepsilon>0, \lambda=(a-b) / \sqrt{2(a+b)} \geq 1+\varepsilon$. Then there exists $\Delta_{\text {est }}=\Delta_{\text {est }}(\varepsilon)>0$ and $d_{*}=d_{*}(\varepsilon)>0$ such that, for all $d \geq d_{*}(\varepsilon)$

$$
\mathbb{P}\left(\frac{1}{n}\left|\left\langle\hat{\boldsymbol{x}}^{S D P}(G), \boldsymbol{x}_{0}\right\rangle\right| \geq \Delta_{e s t}(\varepsilon)\right) \geq 1-C e^{-n^{1 / 2} / C},
$$

with $\mathbb{P}(\cdot)$ denoting expectation with respect to the algorithm and the graph $G$, and $C=C(\varepsilon)$ a constant.

\subsection{Robustness}

Consider the problem of testing whether the graph $G$ has a community structure, i.e. whether $G \sim \mathrm{G}(n, a / n, b / n)$ or $G \sim \mathrm{G}(n, d / n), d=(a+b) / 2$. The next result establishes that the SDP-based test of Section 1.3 is robust with respect to adversarial perturbations of these models. Namely, an adversary can arbitrarily modify $o(n)$ edges of these graphs, without changing the detection threshold.

Corollary 4.1. Let $\mathbb{P}_{0}$ the law of $G \sim \mathrm{G}(n, d / n)$, and $\mathbb{P}_{1}$ be the law of $G \sim \mathrm{G}(n, a / n, b / n)$. Denote by $\widetilde{\mathbb{P}}_{0}, \widetilde{\mathbb{P}}_{1}$ be any two distributions over graphs with vertex set $V=[n]$. Assume that, for each $a \in\{0,1\}$, the following happens: there exists a coupling $\mathbb{Q}_{a}$ of $\mathbb{P}_{a}$ and $\widetilde{\mathbb{P}}_{a}$ such that, if $(G, \widetilde{G}) \sim$ $\mathbb{Q}_{a}$, then $|E(G) \triangle E(\widetilde{G})|=o(n)$ with high probability.

Then, under the same assumptions of Theorem 3, the SDP-based test (9) distinguishes $\widetilde{\mathbb{P}}_{0}$ from $\widetilde{\mathbb{P}}_{1}$ with error probability vanishing as $n \rightarrow \infty$.

By comparison, spectral methods such as the one of [13] appear to be fragile to an adversarial perturbation of $o(n)$ edges [39].

\subsection{Multiple Communities}

The hidden partition model of Eq. (2) can be naturally generalized to the case of $r>2$ hidden communities. Namely, we define the distribution $\mathrm{G}_{r}(n, a / n, b / n)$ over graphs as follows. The vertex set $[n]$ is partitioned uniformly at random into $r$ subsets $S_{1}, S_{2}, \ldots, S_{r}$ with $\left|S_{i}\right|=n / r$. Conditional on this partition, edges are independent with

$\mathbb{P}_{1}\left((i, j) \in E \mid\left\{S_{\ell}\right\}_{\ell \leq r}\right)= \begin{cases}a / n & \text { if }\{i, j\} \subseteq S_{\ell} \text { for an } \ell \in[r] \\ b / n & \text { otherwise. }\end{cases}$ 
The resulting graph has average degree $d=[a+(r-1) b] / r$. The case studied above (hidden bisection) is recovered by setting $r=2$ in this definition: $\mathrm{G}(n, a / n, b / n)=\mathrm{G}_{2}(n, a / n, b / n)$. Of course, this model can be generalized further by allowing for $r$ unequal subsets, and a generic $r \times r$ matrix of edge probabilities [37, 2, 36].

Given a single realization of the graph $G$, we would like to test whether $G \sim \mathrm{G}(n, d / n)$ (hypothesis 0 ), or $G \sim$ $\mathrm{G}_{r}(n, a / n, b / n)$ (hypothesis 1$)$. We use the same SDP relaxation already introduced in Eq. (4), and the test $T(\cdot ; \delta)$ defined in Eq. (9). This is particularly appealing because it does not require knowledge of the number of communities $r$.

TheOREM 7. Consider the problem of distinguishing $G \sim$ $\mathrm{G}_{r}(n, a / n, b / n)$ from $G \sim \mathrm{G}(n, d / n), d=(a+(r-1) b) / r$. Assume, for some $\varepsilon>0$,

$$
\frac{a-b}{\sqrt{r(a+(r-1) b)}} \geq 1+\varepsilon .
$$

Then there exists $\delta_{*}=\delta_{*}(\varepsilon, r)>0$ and $d_{*}=d_{*}(\varepsilon, r)>0$ such that the following holds. If $d \geq d_{*}$, then the SDPbased test $T\left(\cdot ; \delta_{*}\right)$ succeeds with error probability probability at most $C e^{-n / C}$ for $C=C(a, b, r)$ a constant.

Remark 8. In earlier work, a somewhat tighter relaxation is sometimes used, including the additional constraint $X_{i j} \geq$ $-(r-1)^{-1}$ for all $i \neq j$. The simpler relaxation used here is however sufficient for proving Theorem 7 .

Remark 9. The threshold established in Theorem 7 coincides (for large degrees) with the one of spectral methods using non-backtracking random walks [13]. However, for $k \geq 4$ there appears to be a gap between general statistical tests and what is achieved by polynomial time algorithms $[23,18]$.

\section{ACKNOWLEDGMENTS}

A.M. was partially supported by NSF grants CCF-1319979 and DMS-1106627 and the AFOSR grant FA9550-13-1-0036. S.S was supported by the William R. and Sara Hart Kimball Stanford Graduate Fellowship.

\section{REFERENCES}

[1] E. Abbe, A. S. Bandeira, and G. Hall. Exact recovery in the stochastic block model. arXiv:1405.3267, 2014.

[2] E. Abbe and C. Sandon. Community detection in general stochastic block models: fundamental limits and efficient recovery algorithms. In Foundations of Computer Science (FOCS), 2015 IEEE 55th Annual Symposium on, 2015.

[3] N. Alon, M. Krivelevich, and B. Sudakov. Finding a large hidden clique in a random graph. Random Structures and Algorithms, 13(3-4):457-466, 1998.

[4] N. Alon, K. Makarychev, Y. Makarychev, and A. Naor. Quadratic forms on graphs. Inventiones mathematicae, 163(3):499-522, 2006.

[5] A. Ambainis, A. Bačkurs, K. Balodis, D. Kravčenko, R. Ozols, J. Smotrovs, and M. Virza. Quantum strategies are better than classical in almost any xor game. In Automata, Languages, and Programming, pages 25-37. Springer, 2012.
[6] G. W. Anderson, A. Guionnet, and O. Zeitouni. An Introduction to Random Matrices. Cambridge studies in advanced mathematics. Cambridge University Press, 2009.

[7] P. Awasthi, A. S. Bandeira, M. Charikar, R. Krishnaswamy, S. Villar, and R. Ward. Relax, no need to round: Integrality of clustering formulations. In Proceedings of the 2015 Conference on Innovations in Theoretical Computer Science, pages 191-200. ACM, 2015.

[8] J. Baik, G. Ben Arous, and S. Péché. Phase transition of the largest eigenvalue for nonnull complex sample covariance matrices. Annals of Probability, pages 1643-1697, 2005.

[9] A. S. Bandeira, M. Charikar, A. Singer, and A. Zhu. Multireference alignment using semidefinite programming. In Proceedings of the 5th conference on Innovations in theoretical computer science, pages 459-470. ACM, 2014.

[10] M. Bayati, D. Gamarnik, and P. Tetali. Combinatorial approach to the interpolation method and scaling limits in sparse random graphs. Annals of Probability, 41(6):4080-4115, 2013.

[11] F. Benaych-Georges, A. Guionnet, and M. Maïda. Large deviations of the extreme eigenvalues of random deformations of matrices. Probability Theory and Related Fields, 154(3-4):703-751, 2012.

[12] A. Bloemendal and B. Virág. Limits of spiked random matrices I. Probability Theory and Related Fields, 156(3-4):795-825, 2013.

[13] C. Bordenave, M. Lelarge, and L. Massoulié. Non-backtracking spectrum of random graphs: community detection and non-regular Ramanujan graphs. In Foundations of Computer Science (FOCS), 2015 IEEE 55th Annual Symposium on, 2015.

[14] S. Boucheron, G. Lugosi, and P. Massart. Concentration Inequalities: A Nonasymptotic Theory of Independence. Oxford University Press, 2013.

[15] J. Briët, F. M. de Oliveira Filho, and F. Vallentin. The positive semidefinite grothendieck problem with rank constraint. In Automata, Languages and Programming, pages 31-42. Springer, 2010.

[16] Briët, Jop and de Oliveira Filho, Fernando Mário and Vallentin, Frank. Grothendieck inequalities for semidefinite programs with rank constraint. arXiv:1011.1754, 2010.

[17] M. Capitaine, C. Donati-Martin, D. Féral, M. Février, et al. Free convolution with a semicircular distribution and eigenvalues of spiked deformations of wigner matrices. Electron. J. Probab, 16(64):1750-1792, 2011.

[18] Y. Chen and J. Xu. Statistical-computational tradeoffs in planted problems and submatrix localization with a growing number of clusters and submatrices. arXiv:1402.1267, 2014.

[19] P. Chin, A. Rao, and V. Vu. Stochastic block model and community detection in the sparse graphs: A spectral algorithm with optimal rate of recovery. arXiv:1501.05021, 2015.

[20] A. Coja-Oghlan. The Lovász number of random graphs. In Approximation, Randomization, and Combinatorial Optimization. Algorithms and Techniques, pages 228-239. Springer, 2003. 
[21] A. Coja-Oghlan. A spectral heuristic for bisecting random graphs. Random Structures \& Algorithms, 29(3):351-398, 2006.

[22] A. Coja-Oghlan. Graph partitioning via adaptive spectral techniques. Combinatorics, Probability and Computing, 19(02):227-284, 2010.

[23] A. Decelle, F. Krzakala, C. Moore, and L. Zdeborová. Asymptotic analysis of the stochastic block model for modular networks and its algorithmic applications. Physical Review E, 84(6):066106, 2011.

[24] A. Dembo, A. Montanari, and S. Sen. Extremal cuts of sparse random graphs. Annalso of Probability, 2016.

[25] U. Feige and E. Ofek. Spectral techniques applied to sparse random graphs. Random Structures \& Algorithms, 27(2):251-275, 2005.

[26] D. Féral and S. Péché. The largest eigenvalue of rank one deformation of large wigner matrices. Communications in mathematical physics, 272(1):185-228, 2007.

[27] S. Franz and M. Leone. Replica bounds for optimization problems and diluted spin systems. $J$. Stat. Phys., 111:535-564, 2003.

[28] S. Franz, M. Leone, and F. L. Toninelli. Replica bounds for diluted non-poissonian spin systems. $J$. Phys. A, 36:10967-10985, 2003.

[29] J. Friedman. A proof of alon's second eigenvalue conjecture. In Proc. of the 35th Symp. on Theory of Computing, San Diego, pages 720-724, 2003.

[30] M. X. Goemans and D. P. Williamson. Improved approximation algorithms for maximum cut and satisfiability problems using semidefinite programming. Journal of the ACM (JACM), 42(6):1115-1145, 1995.

[31] D. Grigoriev. Linear lower bound on degrees of positivstellensatz calculus proofs for the parity. Theoretical Computer Science, 259(1):613-622, 2001.

[32] A. Grothendieck. Résumé de la théorie métrique des produits tensoriels topologiques. Resenhas do Instituto de Matemática e Estatística da Universidade de São Paulo, 2(4):401-481, 1996.

[33] O. Guédon and R. Vershynin. Community detection in sparse networks via grothendieckâÁŹs inequality. Probability Theory and Related Fields, pages 1-25, 2015.

[34] F. Guerra and F. L. Toninelli. The high temperature region of the Viana-Bray diluted spin glass models. $J$. Stat. Phys, 115:531-555, 2004.

[35] B. Hajek, Y. Wu, and J. Xu. Achieving exact cluster recovery threshold via semidefinite programming. arXiv:1412.6156, 2014.

[36] B. Hajek, Y. Wu, and J. Xu. Achieving exact cluster recovery threshold via semidefinite programming: Extensions. arXiv:1502.07738, 2015.

[37] P. W. Holland, K. Laskey, and S. Leinhardt. Stochastic blockmodels: First steps. Social Networks, 5(2):109-137, 1983.

[38] S. Janson, T. Luczak, and A. Rucinski. Random Graphs. John Wiley and Sons., 2000.

[39] A. Javanmard, A. Montanari, and F. Ricci-Tersenghi. Phase transitions in semidefinite relaxations. In press, 2016.
[40] R. H. Keshavan, A. Montanari, and S. Oh. Matrix completion from noisy entries. Journal of Machine Learning Research, 11:2057-2078, 2010.

[41] A. Knowles and J. Yin. The isotropic semicircle law and deformation of wigner matrices. Communications on Pure and Applied Mathematics, 2013.

[42] A. Kolla, K. Makarychev, and Y. Makarychev. How to play unique games against a semi-random adversary: Study of semi-random models of unique games. In Foundations of Computer Science (FOCS), 2011 IEEE 52nd Annual Symposium on, pages 443-452. IEEE, 2011.

[43] M. Krivelevich and B. Sudakov. The largest eigenvalue of sparse random graphs. Combinatorics, Probability and Computing, 12(01):61-72, 2003.

[44] F. Krzakala, C. Moore, E. Mossel, J. Neeman, A. Sly, L. Zdeborová, and P. Zhang. Spectral redemption in clustering sparse networks. Proceedings of the National Academy of Sciences, 110(52):20935-20940, 2013.

[45] C. M. Le and R. Vershynin. Concentration and regularization of random graphs. arXiv:1506.00669, 2015.

[46] J. W. Lindeberg. Eine neue herleitung des exponentialgesetzes in der wahrscheinlichkeitsrechnung. Mathematische Zeitschrift, 15(1):211-225, 1922.

[47] L. Massoulié. Community detection thresholds and the weak Ramanujan property. In Proceedings of the 46th Annual ACM Symposium on Theory of Computing, pages 694-703. ACM, 2014.

[48] F. McSherry. Spectral partitioning of random graphs. In Foundations of Computer Science, 2001. Proceedings. 42nd IEEE Symposium on, pages 529-537. IEEE, 2001.

[49] A. Megretski. Relaxations of quadratic programs in operator theory and system analysis. In Systems, approximation, singular integral operators, and related topics, pages 365-392. Springer, 2001.

[50] A. Moitra, W. Perry, and A. S. Wein. How robust are reconstruction thresholds for community detection? arXiv:1511.01473, 2015.

[51] E. Mossel, J. Neeman, and A. Sly. Stochastic block models and reconstruction. arXiv:1202.1499, 2012.

[52] E. Mossel, J. Neeman, and A. Sly. A proof of the block model threshold conjecture. arXiv:1311.4115, 2013.

[53] A. Nemirovski, C. Roos, and T. Terlaky. On maximization of quadratic form over intersection of ellipsoids with common center. Mathematical Programming, 86(3):463-473, 1999.

[54] A. Y. Ng, M. I. Jordan, Y. Weiss, et al. On spectral clustering: Analysis and an algorithm. Advances in neural information processing systems, 2:849-856, 2002.

[55] D. Panchenko and M. Talagrand. Bounds for diluted mean-fields spin glass models. Probability Theory and Related Fields, 130(3):319-336, 2004.

[56] A. Pizzo, D. Renfrew, and A. Soshnikov. On finite rank deformations of wigner matrices. Annales de l'Institut Henri Poincaré, Probabilités et Statistiques, 49(1):64-94, 2013.

[57] G. Schoenebeck. Linear level Lasserre lower bounds 
for certain k-CSPs. In Foundations of Computer Science, 2008. FOCS'08. IEEE 49th Annual IEEE Symposium on, pages 593-602. IEEE, 2008.

\section{APPENDIX}

\section{A. PROOFS OF THEOREM 1 AND THEO- REM 3 (MAIN THEOREMS)}

In this Section we prove Theorem 1 and Theorem 3 using Theorems 4, 5 and Lemmas 3.1, 3.2. The proof of Theorem 5 is outlined in Appendices B, C. The others are omitted because of space limitations.

We begin by proving a general approximation result, and then obtain Theorem 1 and Theorem 3 as consequences.

\section{A.1 Three Technical Lemmas}

Lemma A.1. Let $G \sim \mathrm{G}(n, a / n, b / n), d=(a+b) / 2$, and $\boldsymbol{A}_{G}^{c e n}=\boldsymbol{A}_{G}-(d / n) \mathbf{1 1}^{\top}$ be its centered adjacency matrix. For $\lambda \in \mathbb{R}$ fixed, define $\boldsymbol{B}=\boldsymbol{B}(\lambda)$ to be the deformed $G O E$ matrix in Eq. (19).

Then, there exists a universal constant $C$ such that, for either $\boldsymbol{M} \in\left\{\boldsymbol{A}_{G}^{c e n} / \sqrt{d}, \boldsymbol{B}(\lambda)\right\}$, for all $t \geq 0$

$$
\mathbb{P}\{|\Phi(\beta, k ; \boldsymbol{M})-\mathbb{E} \Phi(\beta, k ; \boldsymbol{M})| \geq n t\} \leq C e^{-n t^{2} / C} .
$$

Proof. Define the following Gibbs probability measure over $\left(\mathbb{S}^{k-1}\right)^{n}$, which is naturally associated to the free energy $\Phi:$

$$
\begin{aligned}
& \mu_{\boldsymbol{M}}(\boldsymbol{\sigma}) \equiv \frac{\exp \left(\beta H_{\boldsymbol{M}}(\boldsymbol{\sigma})\right)}{\int \exp \left(\beta H_{\boldsymbol{M}}(\boldsymbol{\tau})\right) \mathrm{d} \nu(\boldsymbol{\tau})} \mathrm{d} \nu(\boldsymbol{\sigma}), \\
& H_{\boldsymbol{M}}(\boldsymbol{\sigma})=\langle\boldsymbol{\sigma}, \boldsymbol{M} \boldsymbol{\sigma}\rangle=\sum_{i, j=1}^{n} M_{i j}\left\langle\boldsymbol{\sigma}_{i}, \boldsymbol{\sigma}_{j}\right\rangle .
\end{aligned}
$$

It is a straightforward exercise with moment generating functions to show that

$$
\frac{\partial \Phi}{\partial M_{i j}}(\beta, k ; \boldsymbol{M})=\mu_{\boldsymbol{M}}\left(\left\langle\boldsymbol{\sigma}_{i}, \boldsymbol{\sigma}_{j}\right\rangle\right),
$$

where $\mu_{M}(f(\boldsymbol{\sigma}))$ denotes the expectation of $f(\boldsymbol{\sigma})$ with respect to the probability measure $\mu_{\boldsymbol{M}}$. In particular, since $\left|\left\langle\boldsymbol{\sigma}_{i}, \boldsymbol{\sigma}_{j}\right\rangle\right| \leq 1$ (here $\|\cdot\|_{2}$ denotes the vector $\ell_{2}$ norm)

$$
\left\|\nabla_{M} \Phi\right\|_{2}^{2}=\sum_{i, j=1}^{n}\left|\frac{\partial \Phi}{\partial M_{i j}}\right|^{2} \leq n^{2} .
$$

This implies Eq. (27) for $\boldsymbol{M}=\boldsymbol{B}$ by Gaussian isoperimetry (with constant $C=4$ ).

For $\boldsymbol{M}=\boldsymbol{A}_{G}^{\mathrm{cen}}$ the proof is analogous. Let $G$ be a graph that does not contain edge $(i, j)$, and $G^{+}$denote the same graph, to which edge $(i, j)$ has been added. Then writing the definition of $\Phi(\cdots)$, we get

$$
\begin{array}{r}
\Phi\left(\beta, k ; \boldsymbol{A}_{G^{+}}^{\text {cen }} / \sqrt{d}\right)-\Phi\left(\beta, k ; \boldsymbol{A}_{G}^{\text {cen }} / \sqrt{d}\right)= \\
\frac{1}{\beta} \log \left\{\mu_{\boldsymbol{A}_{G}^{\mathrm{cen}}}\left(e^{\frac{\beta}{\sqrt{d}}\left\langle\boldsymbol{\sigma}_{i}, \boldsymbol{\sigma}_{j}\right\rangle}\right)\right\} .
\end{array}
$$

In particular

$$
\left|\Phi\left(\beta, k ; \boldsymbol{A}_{G^{+}}^{\mathrm{cen}}\right)-\Phi\left(\beta, k ; \boldsymbol{A}_{G}^{\mathrm{cen}}\right)\right| \leq \frac{1}{\sqrt{d}} .
$$

The claim then follows from a standard application of the 'method of bounded differences' [14] i.e. from Azuma-Höeffding inequality, whereby we construct a bounded differences martingale with a number of steps equal to a sufficiently large constant times the number of edges, e.g. $10 d n$.

Lemma A.2. Let $G \sim \mathrm{G}(n, a / n, b / n), d=(a+b) / 2$, and $\boldsymbol{A}_{G}^{c e n}=\boldsymbol{A}_{G}-(d / n) \mathbf{1 1}^{\top}$ be its centered adjacency matrix. Then there exists a universal constant $C$ such that, for any $t \geq 0$

$$
\mathbb{P}\left\{\left|\operatorname{SDP}\left(\boldsymbol{A}_{G}^{c e n}\right)-\mathbb{E} \operatorname{SDP}\left(\boldsymbol{A}_{G}^{c e n}\right)\right| \geq n t\right\} \leq C e^{-n t^{2} /(C d)} .
$$

Proof. Let $G$ be a graph that does not contain edge $(i, j)$, and $G^{+}$denote the same graph, to which edge $(i, j)$ has been added. Let $\boldsymbol{X} \in \mathrm{PSD}_{1}(n)$ be an optimizer of the SDP with data $\boldsymbol{A}_{G}^{\text {cen }}$, i.e. a feasible point such that $\left\langle\boldsymbol{A}_{G}^{\text {cen }}, \boldsymbol{X}\right\rangle=$ $\operatorname{SDP}\left(\boldsymbol{A}_{G}^{\mathrm{cen}}\right)$. Then

$$
\begin{aligned}
\operatorname{SDP}\left(\boldsymbol{A}_{G^{+}}^{\text {cen }}\right) & \geq\left\langle\boldsymbol{A}_{G^{+}}^{\text {cen }}, \boldsymbol{X}\right\rangle \\
& =\left\langle\boldsymbol{A}_{G}^{\text {cen }}, \boldsymbol{X}\right\rangle+X_{i j} \\
& \geq \operatorname{SDP}\left(\boldsymbol{A}_{G}^{\text {cen }}\right)-1,
\end{aligned}
$$

where we used the fact that $\boldsymbol{X}$ is positive semidefinite to obtain $\left|X_{i j}\right| \leq \sqrt{X_{i i} X_{j j}}=1$. Exchanging the role of $G$ and $G^{+}$, we obtain

$$
\left|\operatorname{SDP}\left(\boldsymbol{A}_{G^{+}}^{\text {cen }}\right)-\operatorname{SDP}\left(\boldsymbol{A}_{G}^{\text {cen }}\right)\right| \leq 1,
$$

As in the previous lemma, the claim follows from an application of the 'method of bounded differences' [14] i.e. from Azuma-Höeffding inequality (we can apply this to a martingale with a number of steps proportional to the expected number of edges, say $10 d n$, whence the claimed probability bound follows).

Lemma A.3. Let $\boldsymbol{A}_{G}^{c e n}, \boldsymbol{B}$ be defined as in Lemma A.1. Then, there exists an absolute constant $C>0$ such that the following holds with probability at least $1-C e^{-n / C}$ :

$$
\left\|\boldsymbol{A}_{G}^{c e n}\right\|_{\infty \rightarrow 2} \leq C d \sqrt{n}, \quad\|\boldsymbol{B}\|_{\infty \rightarrow 2} \leq(C+\lambda) \sqrt{n}
$$

Proof. For $\boldsymbol{B}$ we use (letting $\|\boldsymbol{M}\|_{2 \rightarrow 2}=\|\boldsymbol{M}\|_{o p}=$ $\left.\max \left(\lambda_{1}(\boldsymbol{M}),-\left|\lambda_{n}(\boldsymbol{M})\right|\right)\right)$ :

$$
\begin{aligned}
\|\boldsymbol{B}\|_{\infty \rightarrow 2} & \leq \sqrt{n}\|\boldsymbol{B}\|_{2 \rightarrow 2} \leq \sqrt{n}\left(\lambda+\|\boldsymbol{W}\|_{2 \rightarrow 2}\right) \\
& \leq(C+\lambda) \sqrt{n},
\end{aligned}
$$

where the last inequality holds with the desired probability by standard concentration bounds on the extremal eigenvalues of GOE matrices [6][Section 2.3].

For $\boldsymbol{A}_{G}^{\text {cen }}$, first note that

$$
\begin{aligned}
\left\|\boldsymbol{A}_{G}^{\mathrm{cen}}\right\|_{\infty \rightarrow 2} & \leq\left\|\boldsymbol{A}_{G}\right\|_{\infty \rightarrow 2}+\frac{d}{n}\left\|\mathbf{1 1}^{\top}\right\|_{\infty \rightarrow 2} \\
& \leq\left\|\boldsymbol{A}_{G}\right\|_{\infty \rightarrow 2}+\frac{d}{\sqrt{n}}\left\|\mathbf{1 1}^{\top}\right\|_{2 \rightarrow 2} \\
& \leq\left\|\boldsymbol{A}_{G}\right\|_{\infty \rightarrow 2}+d \sqrt{n} .
\end{aligned}
$$

Next we observe that $\boldsymbol{\sigma} \mapsto\left\|\boldsymbol{A}_{G} \boldsymbol{\sigma}\right\|_{2}^{2}$ is a convex function on $\|\boldsymbol{\sigma}\|_{\infty} \leq 1$, and thus attains it maxima at one of the corners of the hypercube $[-1,1]^{n}$. In other words, $\left\|\boldsymbol{A}_{G}\right\|_{\infty \rightarrow 2}^{2}=$ $\max _{\boldsymbol{\sigma} \in\{ \pm 1\}^{n}}\left\|\boldsymbol{A}_{G} \boldsymbol{\sigma}\right\|_{2}^{2}$. For $\boldsymbol{\sigma} \in\{+1,-1\}^{n}$, we get

$$
\left\|\boldsymbol{A}_{G} \boldsymbol{\sigma}\right\|_{2}^{2} \leq \sum_{i=1}^{n} \operatorname{deg}_{G}(i)^{2}
$$

where $\operatorname{deg}_{G}(i)$ is the degree of vertex $i$ in $G$. The desired bound follows since $\sum_{i=1}^{n} \operatorname{deg}(i)^{2} \leq C_{0} d^{2} n$ with the desired probability for some constant $C_{0}$ large enough (see, e.g. [38]). 


\section{A.2 A General Approximation Result}

TheOREM 8. Let $G \sim \mathrm{G}(n, a / n, b / n), d=(a+b) / 2$, and $\boldsymbol{A}_{G}^{c e n}=\boldsymbol{A}_{G}-(d / n) \mathbf{1 1}^{\top}$ be its centered adjacency matrix. Let $\lambda=(a-b) / \sqrt{2(a+b)}$ and define $\boldsymbol{B}=\boldsymbol{B}(\lambda)$ to be the deformed GOE matrix in Eq. (19). Then, there exists $C=C(\lambda)$ such that, with probability at least $1-C e^{-n / C}$, for all $n \geq n_{0}(a, b)$

$$
\begin{aligned}
\left|\frac{1}{n \sqrt{d}} \operatorname{SDP}\left(\boldsymbol{A}_{G}^{c e n}\right)-\frac{1}{n} \operatorname{SDP}(\boldsymbol{B}(\lambda))\right| & \leq \frac{C \log d}{d^{1 / 10}}, \\
\left|\frac{1}{n \sqrt{d}} \operatorname{SDP}\left(-\boldsymbol{A}_{G}^{c e n}\right)-\frac{1}{n} \operatorname{SDP}(-\boldsymbol{B}(\lambda))\right| & \leq \frac{C \log d}{d^{1 / 10}} .
\end{aligned}
$$

Further $C(\lambda)$ is bounded over compact intervals $\lambda \in\left[0, \lambda_{\max }\right]$

Proof. Throughout the proof $C=C(\lambda)$ is a constant that depends uniquely on $\lambda$, bounded as in the statement, and we will write 'for $n$ large enough' whenever a statement holds for $n \geq n_{0}(a, b)$.

First notice that by Lemma 3.2 and Lemma A.1 we have, with probability larger than $1-C e^{-n / C}$, and all $n$ large enough,

$$
\mid \frac{1}{n} \Phi\left(\beta, k ; \boldsymbol{A}_{G}^{\mathrm{cen}} / \sqrt{d}\right)-\frac{1}{n} \Phi\left((\beta, k ; \boldsymbol{B}(\lambda)) \mid \leq \frac{4 \beta^{2}}{\sqrt{d}}+\frac{10 \lambda^{1 / 2}}{d^{1 / 4}} .\right.
$$

Next, by Lemma 3.1 and Lemma A.3, with the same probability, for $\boldsymbol{M} \in\left\{\boldsymbol{A}_{G}^{\text {cen }} / \sqrt{d}, \boldsymbol{B}(\lambda)\right\}$, and $\beta, d>1$

$$
\left|\frac{1}{n} \Phi(\beta, k ; \boldsymbol{M})-\frac{1}{n} \mathrm{OPT}_{k}(\boldsymbol{M})\right| \leq \frac{k}{\beta} \log \left(\frac{C \beta(d+\lambda)}{k}\right)
$$

(where we optimized the bound of Lemma 3.1 over $\varepsilon$.) Using triangle inequality with Eq. (48), and optimizing over $\beta$, we get, always with probability at least $1-C e^{-n / C}$,

$$
\left|\frac{1}{n \sqrt{d}} \mathrm{OPT}_{k}\left(\boldsymbol{A}_{G}^{\text {cen }}\right)-\frac{1}{n} \mathrm{OPT}_{k}(\boldsymbol{B}(\lambda))\right| \leq \frac{C k^{2 / 3}}{d^{1 / 6}} \log (d+\lambda) .
$$

Proceeding the same way (with $\beta$ replaced by $-\beta$ ), we also obtain

$\left|\frac{1}{n \sqrt{d}} \mathrm{OPT}_{k}\left(-\boldsymbol{A}_{G}^{\mathrm{cen}}\right)-\frac{1}{n} \mathrm{OPT}_{k}(-\boldsymbol{B}(\lambda))\right| \leq \frac{C k^{2 / 3}}{d^{1 / 6}} \log (d+\lambda)$.

Since $\left|\mathrm{OPT}_{k}(-\boldsymbol{B})\right|,\left|\mathrm{OPT}_{k}(-\boldsymbol{B})\right| \leq n\|\boldsymbol{B}\|_{o p} \leq C n$ with probability at least $1-C e^{-n / C}$, we get also

$$
\max \left\{\frac{1}{n} \mathrm{OPT}_{k}( \pm \boldsymbol{B}), \frac{1}{n \sqrt{d}} \mathrm{OPT}_{k}\left( \pm \boldsymbol{A}_{G}^{\mathrm{cen}}\right)\right\} \leq C,
$$

whence, using Theorem 4, we obtain

$$
\begin{aligned}
\left|\frac{1}{n \sqrt{d}} \operatorname{SDP}\left(\boldsymbol{A}_{G}^{\mathrm{cen}}\right)-\frac{1}{n \sqrt{d}} \mathrm{OPT}_{k}\left(\boldsymbol{A}_{G}^{\mathrm{cen}}\right)\right| & \leq \frac{C}{k}, \\
\left|\frac{1}{n} \operatorname{SDP}(\boldsymbol{B})-\frac{1}{n} \mathrm{OPT}_{k}(\boldsymbol{B})\right| & \leq \frac{C}{k} .
\end{aligned}
$$

The claim (46) follows from using this, together with Eq. (50) and triangular inequality. Equation (47) follows from exactly the same argument.

\section{A.3 Proofs of Theorem 1}

Applying Theorem 5 to $\lambda=0$ (whence $\boldsymbol{B}(\lambda)=\boldsymbol{W} \sim$ $\operatorname{GOE}(n))$, we get, with high probability,

$$
\frac{1}{n} \operatorname{SDP}(\boldsymbol{W}), \frac{1}{n} \operatorname{SDP}(-\boldsymbol{W}) \in\left[2-d^{-1}, 2+d^{-1}\right] .
$$

(The claim for $-\boldsymbol{W}$ follows because $-\boldsymbol{W} \sim \operatorname{GOE}(n)$ ) Using Theorem 8, applied to $a=b=d$ (whence $G \sim \mathrm{G}(n, d / n)$ ), we have, with high probability

$$
\begin{array}{r}
\frac{1}{n \sqrt{d}} \operatorname{SDP}\left(\boldsymbol{A}_{G}^{\mathrm{cen}}\right), \frac{1}{n \sqrt{d}} \operatorname{SDP}\left(-\boldsymbol{A}_{G}^{\mathrm{cen}}\right) \in \mathcal{J}(d), \\
\mathcal{J}(d) \equiv\left[2-\frac{C \log d}{d^{1 / 10}}, 2+\frac{C \log d}{d^{1 / 10}}\right] .
\end{array}
$$

This implies that desired claim (5) holds with high probability. By the concentration lemma A.2 (with $a=b=d$ ) it also holds with probability at least $1-C(d) e^{-n / C(d)}$.

\section{A.4 Proofs of Theorem 3}

Recall -throughout the proof-that $\lambda=(a-b) / \sqrt{2(a+b)} \geq$ $1+\varepsilon$ and $d=(a+b) / 2$. Further, without loss of generality, we can assume $\lambda \in\left[0, \lambda_{\max }\right]$ with $\lambda_{\max }>1$ fixed (e.g. $\left.\lambda_{\max }=10^{3}\right)$.

Recall that $\mathbb{P}_{0}$ denotes the law of $G \sim \mathrm{G}(n, d / n)$ and $\mathbb{P}_{1}$ the law of $G \sim \mathrm{G}(n, a / n, b / n)$. We can control the probability of false positives (i.e. declaring $G$ to have a twocommunities structure, which it has not) using Theorem 1. For any $\delta>0$, we have

$$
\begin{array}{r}
\lim _{n \rightarrow \infty} \mathbb{P}_{0}(T(G ; \delta)=1)= \\
=\lim _{n \rightarrow \infty} \mathbb{P}_{0}\left(\frac{1}{n} \operatorname{SDP}\left(\boldsymbol{A}_{G}^{\text {cen }}\right) \geq 2(1+\delta) \sqrt{d}\right)=0,
\end{array}
$$

where the last equality holds for any $d \geq d_{0}(\delta)$.

We next bound the probability of false negatives. Let $\Delta(\cdot)$ as per Theorem 5 . By Theorem 8 , there exists $d_{0}^{\prime}=$ $d_{0}^{\prime}(\varepsilon)$ such that, for all $d \geq d_{0}^{\prime}(\varepsilon)$, with high probability for $G \sim \mathrm{G}(n, a / n, b / n)$,

$$
\begin{aligned}
\frac{1}{n \sqrt{d}} \operatorname{SDP}\left(\boldsymbol{A}_{G}^{\text {cen }}\right) & \geq \frac{1}{n} \operatorname{SDP}(\boldsymbol{B}(\lambda))-\frac{1}{4} \Delta(1+\varepsilon) \\
& \geq \frac{1}{n} \operatorname{SDP}(\boldsymbol{B}(1+\varepsilon))-\frac{1}{4} \Delta(1+\varepsilon) \\
& \geq 2+\frac{3}{4} \Delta(1+\varepsilon),
\end{aligned}
$$

where the second inequality follows because $\operatorname{SDP}(\boldsymbol{B}(\lambda))$ is monotone non-decreasing in $\lambda$ and the last inequality follows from Theorem 5 .

Selecting $\delta_{*}(\varepsilon)=\Delta(1+\varepsilon) / 2>0$, we then have

$$
\begin{aligned}
& \lim _{n \rightarrow \infty} \mathbb{P}_{1}\left(T\left(G ; \delta_{*}(\varepsilon)\right)=0\right)= \\
& =\lim _{n \rightarrow \infty} \mathbb{P}_{1}\left(\frac{1}{n \sqrt{d}} \operatorname{SDP}\left(\boldsymbol{A}_{G}^{\text {cen }}\right)<2\left(1+\delta_{*}(\varepsilon)\right)\right) \\
& =\lim _{n \rightarrow \infty} \mathbb{P}_{1}\left(\frac{1}{n \sqrt{d}} \operatorname{SDP}\left(\boldsymbol{A}_{G}^{\text {cen }}\right)<2+\Delta_{*}(1+\varepsilon)\right)=0,
\end{aligned}
$$

where the last equality follows from Eq. (61).

We proved therefore that the error probability vanishes as $n \rightarrow \infty$, provided $d>d_{*}(\varepsilon)=\max \left(d_{0}\left(\delta_{*}(\varepsilon)\right), d_{0}^{\prime}(\varepsilon)\right)$. In 
fact, our argument also implies (eventually adjusting $d_{*}$ )

$$
\begin{gathered}
\lim _{n \rightarrow \infty} \mathbb{P}_{0}\left(\frac{1}{n \sqrt{d}} \operatorname{SDP}\left(\boldsymbol{A}_{G}^{\mathrm{cen}}\right) \geq 2+\frac{\delta_{*}}{2}\right)=0, \\
\lim _{n \rightarrow \infty} \mathbb{P}_{1}\left(\frac{1}{n \sqrt{d}} \operatorname{SDP}\left(\boldsymbol{A}_{G}^{\mathrm{cen}}\right) \leq 2+\delta_{*}\right)=0 .
\end{gathered}
$$

It then follows from the concentration lemma A.2 that these probabilities (and hence the error probability of our test) are bounded by $C e^{-n / C}$ for $C=C(a, b)$ a constant.

\section{B. PROOF OF THEOREM 5. $(A)$ (DEFORMED GOE MATRICES, $\lambda \leq 1$ )}

In this section we prove part $(a)$ of Theorem 5 . We start with two useful technical facts, and then present the actual proof. Throughout $\boldsymbol{B}(\lambda)=(\lambda / n) \mathbf{1 1 ^ { \top }}+\boldsymbol{W}$, with $\boldsymbol{W} \sim$ $\operatorname{GOE}(n)$ is defined as per Eq. (19).

\section{B.1 Two Technical Lemmas}

Lemma B.1. For any fixed $\boldsymbol{W}$, the function $\lambda \mapsto \operatorname{SDP}(\boldsymbol{B}(\lambda))$ is monotone nondecreasing.

Proof. Let $\lambda_{1} \leq \lambda_{2}$ and choose $\boldsymbol{X}_{*} \in \operatorname{PSD}_{1}(n)$ such that $\left\langle\boldsymbol{B}\left(\lambda_{1}\right), \boldsymbol{X}_{*}\right\rangle=\operatorname{SDP}\left(\boldsymbol{B}\left(\lambda_{1}\right)\right)$ (this exists since $\mathrm{PSD}_{1}(n)$ is compact). Then

$$
\begin{aligned}
\operatorname{SDP}\left(\boldsymbol{B}\left(\lambda_{2}\right)\right) & \geq\left\langle\boldsymbol{B}\left(\lambda_{2}\right), \boldsymbol{X}_{*}\right\rangle \\
& \geq\left\langle\boldsymbol{B}\left(\lambda_{1}\right)+\left(\lambda_{2}-\lambda_{1}\right) \mathbf{1 1}^{\top} / n, \boldsymbol{X}_{*}\right\rangle \\
& \geq \operatorname{SDP}\left(\boldsymbol{B}\left(\lambda_{1}\right)\right),
\end{aligned}
$$

where the last inequality follows since $\boldsymbol{X}_{*} \succeq 0$.

Lemma B.2. Fix $\delta \in(0,1]$ and $k(n)=\lfloor n \delta\rfloor$. Let $\boldsymbol{U} \in$ $\mathbb{R}^{n \times k(n)}$ be a uniformly random (Haar measure) orthogonal matrix (in particular $\boldsymbol{U}^{\top} \boldsymbol{U}=\mathrm{I}_{k(n)}$ ). Then there exists $C=$ $C(\delta)$ such that, for any fixed basis vector $\boldsymbol{e}_{i}$,

$$
\mathbb{P}\left(\max _{1 \leq i \leq n}|| \boldsymbol{U}^{\top} \boldsymbol{e}_{i} \|_{2}^{2}-\delta \mid \geq C \sqrt{\frac{\log n}{n}}\right) \geq 1-\frac{1}{n^{20}} .
$$

Proof. In order to lighten the notation, we can assume $n \delta$ to be an integer.

Let $\boldsymbol{P}=\boldsymbol{U} \boldsymbol{U}^{\top}$ be the orthogonal projector on the column space of $\boldsymbol{U}$. By the invariance of the Haar measure under rotations, this is a projector onto a uniformly random subspace of $n \delta$ dimension in $\mathbb{R}^{n}$, and $Y_{i} \equiv\left\|\boldsymbol{U}^{\top} \boldsymbol{e}_{i}\right\|_{2}^{2}=$ $\left\langle\boldsymbol{e}_{i}, \boldsymbol{P} \boldsymbol{e}_{i}\right\rangle=\left\|\boldsymbol{P} \boldsymbol{e}_{i}\right\|_{2}^{2}$. Inverting the role of $\boldsymbol{P}$ and $\boldsymbol{e}_{i}$, we see that $Y_{i i}$ is distributed as the square norm of the first $n \delta$ components of a uniformly random unit vector of $n$ dimensions. Hence

$$
Y_{i} \stackrel{\mathrm{d}}{=} \frac{Z_{n \delta}}{Z_{n \delta}+Z_{n(1-\delta)}},
$$

where $Z_{\ell} \sim \chi^{2}(\ell), \ell \in\{n \delta, n(1-\delta)\}$ denote two independent chi-squared random variable with $\ell$ degrees of freedom. Standard tail bounds on chi-squared random variables imply the claim.

\section{B.2 Proof of Theorem 5. (a)}

We first note that

$$
\frac{1}{n} \operatorname{SDP}(\boldsymbol{B}(\lambda)) \leq \xi_{1}(\boldsymbol{B}(\lambda)) \leq 2+o_{n}(1),
$$

where the last inequality holds with high probability, by, e.g., [41][Theorem 2.7].

It is therefore sufficient to prove that, for any $\varepsilon>0$, $\operatorname{SDP}(\boldsymbol{B}(\lambda)) / n \geq 2-\varepsilon$ with probability converging to one as $n \rightarrow \infty$. By Lemma B.1, we only need to prove this for $\lambda=$ 0 , i.e. to lower bound $\operatorname{SDP}(\boldsymbol{W})$ for $\boldsymbol{W} \sim \operatorname{GOE}(n)$. We will achieve this by constructing a witness, i.e. a feasible point $\boldsymbol{X} \in \mathrm{PSD}_{1}(n)$, depending on $\boldsymbol{W}$ such that $\langle\boldsymbol{W}, \boldsymbol{X}\rangle / n \geq 2-\varepsilon$ with high probability.

A more general construction will be developed in Appendix $\mathrm{C}$ to prove part $(b)$ of the Theorem. The case $\lambda=0$ is however much simpler and we prefer to present it separately here to build intuition.

Fix $\delta>0$, and let $\boldsymbol{u}_{1}, \boldsymbol{u}_{2}, \ldots \boldsymbol{u}_{n \delta}$ be the eigenvectors of $\boldsymbol{W}$ corresponding to the top $n \delta$ eigenvalues. Denote by $\boldsymbol{U} \in$ $\mathbb{R}^{n \times(n \delta)}, \boldsymbol{U}^{\top} \boldsymbol{U}=\mathrm{I}_{n \delta}$ the matrix whose columns are $\boldsymbol{u}_{1}$, $\boldsymbol{u}_{2}, \ldots \boldsymbol{u}_{n \delta}$, and let $\boldsymbol{D} \in \mathbb{R}^{n \times n}$ be the diagonal matrix with entries

$$
\boldsymbol{D}_{i i}=\left(\boldsymbol{U} \boldsymbol{U}^{\top}\right)_{i i}
$$

Note that, by invariance of the GOE distribution under orthogonal transformations, $\boldsymbol{U}$ is a uniformly random orthogonal matrix. Hence by Lemma B.2 and union bound

$$
\mathbb{P}\left(\max _{i \in[n]}\left|D_{i i}-\delta\right| \leq C \sqrt{\frac{\log n}{n}}\right) \geq 1-\frac{1}{n^{9}},
$$

for $C=C(\delta)$ a suitable constant.

We then define our witness as

$$
\boldsymbol{X}=\boldsymbol{D}^{-1 / 2} \boldsymbol{U} \boldsymbol{U}^{\top} \boldsymbol{D}^{-1 / 2} .
$$

Clearly $\boldsymbol{X} \in \mathrm{PSD}_{1}(\boldsymbol{W})$ is a feasible point. Further, letting $\boldsymbol{E}=\delta^{1 / 2} \boldsymbol{D}^{-1 / 2}$

$$
\begin{aligned}
\langle\boldsymbol{W}, \boldsymbol{X}\rangle & =\frac{1}{\delta}\left\langle\boldsymbol{W}, \boldsymbol{U} \boldsymbol{U}^{\top}\right\rangle-\frac{1}{\delta}\left\langle\boldsymbol{W}-\boldsymbol{E} \boldsymbol{W} \boldsymbol{E}, \boldsymbol{U} \boldsymbol{U}^{\top}\right\rangle \\
& \geq \frac{1}{\delta} \sum_{\ell=1}^{n \delta} \xi_{\ell}(\boldsymbol{W})-\frac{1}{\delta}\|\boldsymbol{W}-\boldsymbol{E} \boldsymbol{W} \boldsymbol{E}\|_{2}\left\|\boldsymbol{U} \boldsymbol{U}^{\top}\right\|_{*} \\
& \geq n \xi_{n \delta}(\boldsymbol{W})-\frac{1}{\delta}\|\boldsymbol{W}\|_{2}\left(1+\|\boldsymbol{E}\|_{2}\right)\|\boldsymbol{E}-\mathrm{I}\|_{2}\left\|\boldsymbol{U} \boldsymbol{U}^{\top}\right\|_{*} .
\end{aligned}
$$

Here $\|\boldsymbol{Z}\|_{*}$ denotes the nuclear norm of $b Z$ (sum of the absolute values of eigenvalues) and in the last inequality we used $\|\boldsymbol{W}-\boldsymbol{E} \boldsymbol{W} \boldsymbol{E}\|_{2} \leq\|\boldsymbol{W}-\boldsymbol{E} \boldsymbol{W}\|_{2}+\|\boldsymbol{E} \boldsymbol{W}-\boldsymbol{E} \boldsymbol{W} \boldsymbol{E}\| \leq$ $\|\boldsymbol{W}\|_{2}\|\boldsymbol{E}-\mathrm{I}\|_{2}+\|\boldsymbol{E}\|_{2}\|\boldsymbol{W}\|_{2}\|\boldsymbol{E}-\mathrm{I}\|_{2}$.

Next, since $\boldsymbol{U} \boldsymbol{U}^{\top}$ is a projector on $n \delta$ dimensions, we have $\left\|\boldsymbol{U} \boldsymbol{U}^{\top}\right\|_{*}=n \delta$, whence

$$
\frac{1}{n}\langle\boldsymbol{W}, \boldsymbol{X}\rangle \geq \lambda_{n \varepsilon}(\boldsymbol{W})-\|\boldsymbol{W}\|_{2}\left(2+\|\boldsymbol{E}-\mathrm{I}\|_{2}\right)\|\boldsymbol{E}-\mathrm{I}\|_{2} .
$$

By Eq. (74), we have $\|\boldsymbol{E}-\mathrm{I}\|_{2} \rightarrow 0$ almost surely, and by a classical result [6], also the following limits hold almost surely

$$
\begin{aligned}
\lim _{n \rightarrow \infty}\|\boldsymbol{W}\|_{2} & =2, \\
\lim _{n \rightarrow \infty} \lambda_{n \delta}(\boldsymbol{W}) & =\xi_{*}(\delta),
\end{aligned}
$$

where $\xi_{*}(\delta) \uparrow 2$ as $\delta \rightarrow 0$. Indeed $\xi_{*}(\delta)$ can be expressed explicitly in terms of Wigner semicircle law, namely, for 
$\delta \in(0,1)$ it is the unique positive solution of the following equation.

$$
\int_{\xi_{*}(\delta)}^{2} \frac{\sqrt{4-x^{2}}}{2 \pi} \mathrm{d} x=\delta .
$$

Substituting in Eq. (79), we get, almost surely (and as consequence in probability)

$$
\lim \inf _{n \rightarrow \infty} \frac{1}{n}\langle\boldsymbol{W}, \boldsymbol{X}\rangle \geq \xi_{*}(\delta) \geq 2-\varepsilon .
$$

where the last inequality holds by taking $\delta$ small enough.

\section{PROOF OF THEOREM 5. $(B)$ (DEFORMED GOE MATRICES, $\lambda>1$ ): OUTLINE}

We begin by recalling the definition of the deformed GOE matrix $\boldsymbol{B}=\boldsymbol{B}(\lambda)$, given in Eq. (19),

$$
\boldsymbol{B} \equiv \frac{\lambda}{n} \mathbf{1 1} \mathbf{1}^{\top}+\boldsymbol{W}
$$

where $\boldsymbol{W} \sim \operatorname{GOE}(n)$, and we denote by $\left(\boldsymbol{u}_{1}, \xi_{1}\right), \ldots,\left(\boldsymbol{u}_{n}, \xi_{n}\right)$ denote the eigenpairs of $\boldsymbol{B}$, namely

$$
\boldsymbol{B} \boldsymbol{u}_{k}=\xi_{k} \boldsymbol{u}_{k}
$$

where $\xi_{1} \geq \xi_{2} \geq \cdots \geq \xi_{n}$.

The proof of Theorem 5.(b) is based on the following construction of a witness $\boldsymbol{X}$, which depends on (small) parameters $\varepsilon, \delta>0$ to be fixed at the end. In order not to complicate the notation un-necessarily, we will assume $n \delta$ to be an integer. Let $R: \mathbb{R} \rightarrow \mathbb{R}$ be a 'capping' function, i.e.

$$
R(x) \equiv \begin{cases}1 & \text { if } x \geq 1 \\ x & \text { if }-1<x<1 \\ -1 & \text { if } x \leq-1\end{cases}
$$

We then define $\varphi \in \mathbb{R}^{n}$ by letting $\varphi_{i} \equiv R\left(\varepsilon \sqrt{n} u_{1, i}\right)$. We also define $\boldsymbol{U} \in \mathbb{R}^{n \times(n \delta)}$ as the matrix whose $i$-th column is $\boldsymbol{u}_{i+1}$ (hence it contains the eigenvector $\boldsymbol{u}_{2}, \ldots \boldsymbol{u}_{n \delta+1}$ ). Note that $\boldsymbol{U}$ is an orthogonal matrix: $\boldsymbol{U}^{\top} \boldsymbol{U}=\mathrm{I}_{n \delta}$. Finally, we define $\boldsymbol{D} \in \mathbb{R}^{n \times n}$ to be a diagonal matrix with entries

$$
D_{i i}=\frac{\sqrt{1-\varphi_{i}^{2}}}{\left\|\boldsymbol{U}^{\top} \boldsymbol{e}_{i}\right\|_{2}} .
$$

Our witness construction is defined as

$$
\boldsymbol{X}=\boldsymbol{\varphi} \varphi^{\top}+\boldsymbol{D} \boldsymbol{U} \boldsymbol{U}^{\top} \boldsymbol{D} .
$$

We analyze this construction through a sequence of lemmas. Throughout we assume the above definitions and the setting of Theorem 5 . We use $C, C_{0}, \ldots$ to denote finite nonrandom universal constants. Without loss of generality, we will also assume $\lambda \in\left(1, C_{0}\right)$ for some $C_{0}>1$.

We start from an elementary fact.

Lemma C.1. There exists a constant $C$ such that

$$
\lim _{n \rightarrow \infty} \mathbb{P}\left(\|\boldsymbol{B}\|_{2} \geq C\right)=0 \text {. }
$$

PRoOF. It follows from triangular inequality that $\|\boldsymbol{B}\|_{2} \leq$ $\lambda+\|\boldsymbol{W}\|_{2}$. Hence the claim follows by standard bounds on the eigenvalues of GOE matrices [6][Theorem 2.1.22].

Lemma C.2. There exists a constant $C>0$ such that, with high probability,

$$
\left|\frac{1}{n}\left\langle\boldsymbol{B}, \boldsymbol{\varphi} \boldsymbol{\varphi}^{\top}\right\rangle-\varepsilon^{2} \xi_{1}\right| \leq C \varepsilon^{4} .
$$

Proof. Define $x-R(x) \equiv \bar{R}(x)$. Further, for a vector $\boldsymbol{x}=\left(x_{1}, x_{2}, \ldots, x_{n}\right)$, we write $\bar{R}(\boldsymbol{x})$ for the vector obtained applying $\bar{R}$ componentwise, i.e.

$$
\bar{R}(\boldsymbol{x})=\left(\bar{R}\left(x_{1}\right), \bar{R}\left(x_{2}\right), \ldots, \bar{R}\left(x_{n}\right)\right) .
$$

We then have

$$
\begin{aligned}
& \quad\left|\frac{1}{n}\left\langle\boldsymbol{B}, \boldsymbol{\varphi} \boldsymbol{\varphi}^{\top}\right\rangle-\varepsilon^{2} \xi_{1}\right|= \\
& \quad=\left|\frac{1}{n}\left\langle\boldsymbol{B}, \boldsymbol{\varphi} \boldsymbol{\varphi}^{\top}\right\rangle-\frac{1}{n}\left\langle\boldsymbol{B},\left(\varepsilon \sqrt{n} \boldsymbol{u}_{1}\right)\left(\varepsilon \sqrt{n} \boldsymbol{u}_{1}\right)^{\top}\right\rangle\right| \\
& \quad \leq \frac{2}{n}\left|\left\langle\left(\varepsilon \sqrt{n} \boldsymbol{u}_{1}\right), \boldsymbol{B} \bar{R}\left(\varepsilon \sqrt{n} \boldsymbol{u}_{1}\right)\right\rangle\right| \\
& +\frac{1}{n}\left|\left\langle\bar{R}\left(\varepsilon \sqrt{n} \boldsymbol{u}_{1}\right), \boldsymbol{B} \bar{R}\left(\varepsilon \sqrt{n} \boldsymbol{u}_{1}\right)\right\rangle\right| \\
& \quad \leq 4\|\boldsymbol{B}\|_{2} \frac{1}{\sqrt{n}}\left\|\bar{R}\left(\varepsilon \sqrt{n} \boldsymbol{u}_{1}\right)\right\|_{2} \max \left(\varepsilon ; \frac{1}{\sqrt{n}}\left\|\bar{R}\left(\varepsilon \sqrt{n} \boldsymbol{u}_{1}\right)\right\|_{2}\right)
\end{aligned}
$$

Note that

$$
\bar{R}(x)^{2}= \begin{cases}(|x|-1)^{2} & \text { if }|x| \geq 1 \\ 0 & \text { if }|x|<1 .\end{cases}
$$

In particular $\bar{R}(x)^{2} \leq x^{6}$ for all $x$. We therefore have

$$
\begin{aligned}
\frac{1}{n}\left\|\bar{R}\left(\varepsilon \sqrt{n} \boldsymbol{u}_{1}\right)\right\|_{2}^{2} & =\frac{1}{n} \sum_{i=1}^{n} \bar{R}\left(\varepsilon \sqrt{n} u_{1, i}\right)^{2} \\
& \leq \frac{\varepsilon^{6}}{n} \sum_{i=1}^{n}\left(\sqrt{n} u_{1, i}\right)^{6}
\end{aligned}
$$

Next we decompose $\boldsymbol{u}_{1}=z_{1}(\mathbf{1} / \sqrt{n})+\sqrt{1-z_{1}^{2}} \boldsymbol{u}_{1}^{\perp}$, where $z_{1}=\left|\left\langle\boldsymbol{u}_{1}, \mathbf{1}\right\rangle\right| / \sqrt{n} \in[0,1]$, and $\left\langle\boldsymbol{u}_{1}^{\perp}, \mathbf{1}\right\rangle=0$. Since $(a+b)^{6} \leq$ $2^{5}\left(a^{6}+b^{6}\right)$, we have

$$
\begin{aligned}
\frac{1}{n}\left\|\bar{R}\left(\varepsilon \sqrt{n} \boldsymbol{u}_{1}\right)\right\|_{2}^{2} & \leq \frac{\varepsilon^{6}}{n} \sum_{i=1}^{n} 32\left(1+\left(\sqrt{n} u_{1, i}^{\perp}\right)^{6}\right) \\
& \leq 32 \varepsilon^{6}\left[1+\frac{1}{n} \sum_{i=1}^{n}\left(\sqrt{n} u_{1, i}^{\perp}\right)^{6}\right] \leq C \varepsilon^{6}
\end{aligned}
$$

where the last inequality holds with high probability for some absolute constant $C$ and all $n \geq n_{0}$, by a law of large numbers for the eigenvectors of deformed Wigner matrices, whose proof is omitted because of space reasons. Using this together with Eq. (89) in Eq. (95) we get

$$
\left|\frac{1}{n}\left\langle\boldsymbol{B}, \boldsymbol{\varphi} \boldsymbol{\varphi}^{\top}\right\rangle-\varepsilon^{2} \xi_{1}\right| \leq C \varepsilon^{3} \max \left(\varepsilon ; C \varepsilon^{3}\right) \leq C^{\prime} \varepsilon^{4},
$$

which completes our proof.

Lemma C.3. Let $\boldsymbol{F} \in \mathbb{R}^{n \times n}$ be a diagonal matrix with entries $F_{i i}=\sqrt{1-\varphi_{i}^{2}}$. Then, there exists a constant $K=$ $K(\delta)$ such that, with high probability,

$$
\left|\frac{1}{n}\left\langle\boldsymbol{B}, \boldsymbol{D} \boldsymbol{U} \boldsymbol{U}^{\top} \boldsymbol{D}\right\rangle-\frac{1}{n \delta}\left\langle\boldsymbol{B}, \boldsymbol{F} \boldsymbol{U} \boldsymbol{U}^{\top} \boldsymbol{F}\right\rangle\right| \leq K(\delta) \sqrt{\frac{\log n}{n}} .
$$

Proof. Define $\boldsymbol{H}$ to be a diagonal matrix with entries 
$H_{i i} \equiv \sqrt{\delta} /\left\|\boldsymbol{U}^{\top} \boldsymbol{e}_{i}\right\|_{2}$. Then by definition $\boldsymbol{D}=\boldsymbol{F} \boldsymbol{H} / \sqrt{\delta}$ and

$$
\begin{aligned}
& \left|\frac{1}{n}\left\langle\boldsymbol{B}, \boldsymbol{D} \boldsymbol{U} \boldsymbol{U}^{\top} \boldsymbol{D}\right\rangle-\frac{1}{n \delta}\left\langle\boldsymbol{B}, \boldsymbol{F} \boldsymbol{U} \boldsymbol{U}^{\top} \boldsymbol{F}\right\rangle\right|= \\
& =\frac{1}{n \delta}\left|\left\langle\boldsymbol{F} \boldsymbol{B} \boldsymbol{F}, \boldsymbol{H} \boldsymbol{U} \boldsymbol{U}^{\top} \boldsymbol{H}\right\rangle-\left\langle\boldsymbol{F} \boldsymbol{B} \boldsymbol{F}, \boldsymbol{U} \boldsymbol{U}^{\top}\right\rangle\right| \\
& \leq \frac{1}{n \delta}\|\boldsymbol{H} \tilde{\boldsymbol{B}} \boldsymbol{H}-\tilde{\boldsymbol{B}}\|_{2}\left\|\boldsymbol{U} \boldsymbol{U}^{\top}\right\|_{*},
\end{aligned}
$$

where $\tilde{\boldsymbol{B}}=\boldsymbol{F} \boldsymbol{B} \boldsymbol{F}$, and we recall that $\|\boldsymbol{M}\|_{*}$ denotes the nuclear norm of matrix $\boldsymbol{M}$. Note that $\|\boldsymbol{F}\|_{2}=\max _{i \in[n]}\left|F_{i i}\right| \leq$ 1, hence by Eq. (89) we have $\|\tilde{\boldsymbol{B}}\|_{2} \leq C$ with high probability. Further, since $\boldsymbol{U} \boldsymbol{U}^{\top}$ is a projector on a space of $n \delta$ dimensions, we have $\left\|\boldsymbol{U} \boldsymbol{U}^{\top}\right\|_{*}=n \delta$. Therefore

$$
\begin{aligned}
& \left|\frac{1}{n}\left\langle\boldsymbol{B}, \boldsymbol{D} \boldsymbol{U} \boldsymbol{U}^{\top} \boldsymbol{D}\right\rangle-\frac{1}{n \delta}\left\langle\boldsymbol{B}, \boldsymbol{F} \boldsymbol{U} \boldsymbol{U}^{\top} \boldsymbol{F}\right\rangle\right| \leq \\
& \quad \leq\|\boldsymbol{H} \tilde{\boldsymbol{B}} \boldsymbol{H}-\tilde{\boldsymbol{B}}\|_{2} \\
& \leq\|\tilde{\boldsymbol{B}}\|_{2}\|\boldsymbol{H}-\mathrm{I}\|\left(2+\|\boldsymbol{H}-\mathrm{I}\|_{2}\right) \\
& \leq C\|\boldsymbol{H}-\mathrm{I}\| \max \left(1 ;\|\boldsymbol{H}-\mathrm{I}\|_{2}\right),
\end{aligned}
$$

where we used $\|\tilde{\boldsymbol{B}}\|_{2} \leq\|\boldsymbol{B}\|_{2}\|\boldsymbol{F}\|_{2}^{2} \leq\|\boldsymbol{B}\|_{2} \leq C$ by Lemma C.1. Note that

$$
\|\boldsymbol{H}-\mathrm{I}\|_{2}=\max _{1 \leq i \leq n}\left|\frac{\sqrt{\delta}}{\left\|\boldsymbol{U}^{\top} \boldsymbol{e}_{i}\right\|_{2}}-1\right| .
$$

The proof is completed by Lemma B.2 and union bound.

Lemma C.4. There exists a finite constant $C>0$ such that, for all $\delta, \varepsilon>0$, we have

$$
\begin{aligned}
& \lim _{n \rightarrow \infty} \mathbb{P}\left(\left\langle\boldsymbol{u}_{i}, \boldsymbol{F} \boldsymbol{B} \boldsymbol{F} \boldsymbol{u}_{i}\right\rangle \geq L(\varepsilon, \delta) \forall i \in\{2, \ldots, n \delta+1\}\right)=1, \\
& L(\varepsilon, \delta) \equiv 2-2 \varepsilon^{2}-C \delta^{2 / 3}-C \varepsilon^{4} .
\end{aligned}
$$

The proof of this lemma is longer that the others, and omitted because of space limitations.

We are now in position to prove Theorem 5.(b).

Proof Proof of Theorem 5.(b). We use the explicit construction in Eq. (88). Note that $\boldsymbol{X} \in \mathrm{PSD}_{1}(n)$. Indeed $\boldsymbol{X} \succeq 0$ as it is the sum of two positive-semidefinite matrices. Further, $X_{i i}=1$, since

$$
\begin{aligned}
\left\langle\boldsymbol{e}_{i}, \boldsymbol{X} \boldsymbol{e}_{i}\right\rangle & =\left|\left\langle\boldsymbol{e}_{i}, \boldsymbol{\varphi}\right\rangle\right|^{2}+\left\|\boldsymbol{U}^{\top} \boldsymbol{D} \boldsymbol{e}_{i}\right\|_{2}^{2} \\
& =\varphi_{i}^{2}+D_{i i}^{2}\left\|\boldsymbol{U}^{\top} \boldsymbol{e}_{i}\right\|_{2}^{2}=1 .
\end{aligned}
$$

We are left with the task of lower bounding the objective value. With high probability

$$
\begin{aligned}
\frac{1}{n}\langle\boldsymbol{B}, \boldsymbol{X}\rangle & =\frac{1}{n}\left\langle\boldsymbol{B}, \boldsymbol{\varphi} \varphi^{\top}\right\rangle+\frac{1}{n}\left\langle\boldsymbol{B}, \boldsymbol{D} \boldsymbol{U} \boldsymbol{U}^{\top} \boldsymbol{D}\right\rangle \\
& \geq \varepsilon^{2} \xi_{1}-C \varepsilon^{4}+\frac{1}{n \delta}\left\langle\boldsymbol{B}, \boldsymbol{F} \boldsymbol{U} \boldsymbol{U}^{\top} \boldsymbol{F}\right\rangle-K(\delta) \sqrt{\frac{\log n}{n}},
\end{aligned}
$$

where we used Lemma C.2, and Lemma C.3. For all $n$ large enough, we can bound the term $\sqrt{(\log n) / n}^{1 / 2}$ by $C \varepsilon^{4}$. Further, by [41][Theorem 2.7], $\xi_{1} \geq\left(\lambda+\lambda^{-1}\right)-C^{\prime} n^{-0.4}$ with high probability. Since $\lambda+\lambda^{-1}>2$, there exists $\Delta_{0}(\lambda)>0$ such that, with high probability

$$
\frac{1}{n}\langle\boldsymbol{B}, \boldsymbol{X}\rangle \geq\left(2+\Delta_{0}(\lambda)\right) \varepsilon^{2}-C \varepsilon^{4}+\frac{1}{n \delta} \sum_{i=2}^{n \delta+1}\left\langle\boldsymbol{u}_{i}, \boldsymbol{F} \boldsymbol{B} \boldsymbol{F} \boldsymbol{u}_{i}\right\rangle .
$$

Now we apply Lemma C.4 to get, with high probability

$$
\begin{aligned}
\frac{1}{n}\langle\boldsymbol{B}, \boldsymbol{X}\rangle & \geq\left(2+\Delta_{0}(\lambda)\right) \varepsilon^{2}-C \varepsilon^{4}+2-2 \varepsilon^{2}-C \delta^{2 / 3}-C \varepsilon^{4} \\
& \geq 2+\Delta_{0}(\lambda) \varepsilon^{2}-2 C \varepsilon^{4}-C \delta^{2 / 3} .
\end{aligned}
$$

Setting $\varepsilon=\sqrt{\Delta_{0}(\lambda) /(4 C)}$ and $\delta=\left[\Delta_{0}(\lambda) /\left(16 C^{2}\right)\right]^{3 / 2}$, we conclude that

$$
\lim _{n \rightarrow \infty} \mathbb{P}\left(\frac{1}{n}\langle\boldsymbol{B}, \boldsymbol{X}\rangle \geq 2+\frac{\Delta_{0}(\lambda)^{2}}{16 C}\right)=1,
$$

which completes the proof of the theorem.

\section{PROOF OF THEOREM 6 (ESTIMATION)}

\section{D.1 A Rounding Lemma}

We will need the following rounding lemma, that is of independent interest. While we state it for general expectations of random variables, we will apply it to finite sums (i.e. expectations with respect to random variables that take finitely many values).

Lemma D.1. For $t \in \mathbb{R}_{>0}$, define $s_{t}: \mathbb{R} \rightarrow\{+1,0,-1\}$ by $s_{t}(x)=1$ if $x \geq t, s_{t}(x)=-1$ if $x \leq-t$, and $s_{t}(x)=0$ otherwise.

Let $X_{0}, Y$ be two random variables with $\mathbb{P}\left(X_{0}=+1\right)=$ $\mathbb{P}\left(X_{0}=-1\right)=1 / 2, \mathbb{E}\left(X_{0} Y\right) \geq \varepsilon>0$ and $\mathbb{E}\left(Y^{2}\right)=1$. Then, there exists $t_{*}$ (repending on the joint law of $X_{0}, Y$ ) such that

$$
\mathbb{E}\left\{X_{0} s_{t_{*}}(Y)\right\} \geq \frac{\varepsilon^{2}}{4} .
$$

Proof. Define $Z=X_{0} Y$. Then the assumptions translate into $\mathbb{E}(Z) \geq \varepsilon$ and $\mathbb{E}\left(Z^{2}\right)=1$, while the claim is equivalent to $\mathbb{E}\left\{s_{t}(Z)\right\} \geq \varepsilon^{2} / 4$ (note indeed that $s_{t}(\cdot)$ is an odd function). Now we have

$$
\begin{aligned}
\varepsilon & \leq \mathbb{E}(Z)=\int_{0}^{\infty}[\mathbb{P}(Z \geq t)-\mathbb{P}(Z \leq-t)] \mathrm{d} t \\
& \leq \int_{0}^{T}[\mathbb{P}(Z \geq t)-\mathbb{P}(Z \leq-t)] \mathrm{d} t \\
& +\frac{1}{T} \int_{T}^{\infty} t[\mathbb{P}(Z \geq t)+\mathbb{P}(Z \leq-t)] \mathrm{d} t \\
& \leq \int_{0}^{T} \mathbb{E}\left\{s_{t}(Z)\right\} \mathrm{d} t+\frac{1}{T} \mathbb{E}\left\{Z^{2}\right\} .
\end{aligned}
$$

Taking $T=2 / \varepsilon$, it follows that

$$
\frac{1}{T} \int_{0}^{T} \mathbb{E}\left\{s_{t}(Z)\right\} \mathrm{d} t \geq \frac{\varepsilon^{2}}{4} .
$$

Since the average of $\mathbb{E}\left\{s_{t}(Z)\right\}$ over the interval $t \in[0, T]$ is at least $\varepsilon^{2} / 4$, then there must exist $t_{*} \in[0, T]$ such that $\mathbb{E}\left\{s_{t_{*}}(Z)\right\} \geq \varepsilon^{2} / 4$.

\section{D.2 Proof of Theorem 6}

Throughout this appendix, the partition $V=S_{1} \cup S_{2}$ is fixed. Note that $G_{1} \sim \mathrm{G}\left(n, a^{\prime} / n, b^{\prime} / n\right)$, and $G_{2} \sim$ $\mathrm{G}\left(n, a^{\prime} \delta_{n} / n, b^{\prime} \delta_{n} / n\right)$, with $a^{\prime}=a /\left(1+\delta_{n}\right), b^{\prime}=b /\left(1+\delta_{n}\right)$. For simplicity of notation, we will use $a$ instead of $a^{\prime}$ and $b$ instead of $b^{\prime}$. Note that this does not change the assumptions because it only implies a $o_{n}(1)$ shift in $a, b$. Also, $G_{1}$ and $G_{2}$ are dependent because they cannot share edges. However, if 
they are sampled independently, they will share, with high probability, only $O(1)$ edges. We will therefeore treat them as independent: the incurred error is negligible.

Setting, by definition, the diagonal entries of $\boldsymbol{A}_{G_{1}}^{\text {cen }}$ to be equal to $\lambda \sqrt{d}$, we have

$$
\frac{1}{\sqrt{d}} \boldsymbol{A}_{G_{1}}^{\text {cen }}=\frac{\lambda}{n} \boldsymbol{x}_{0} \boldsymbol{x}_{0}^{\top}+\boldsymbol{E},
$$

where $\boldsymbol{E}=\boldsymbol{E}^{\top}$ has zero mean, $\mathbb{E}\{\boldsymbol{E}\}=0$, with $E_{i i}=0$, and $\left(E_{i j}\right)_{i<j}$ independent

$$
E_{i j}= \begin{cases}\frac{1}{\sqrt{d}}\left(1-\frac{d}{n}\right) & \text { with probability } p_{i j}, \\ -\frac{\sqrt{d}}{n} & \text { with probability } 1-p_{i j} .\end{cases}
$$

Here $p_{i j}=a / n$ if $\{i, j\} \subseteq S_{1}$ or $\{i, j\} \subseteq S_{2}$, and $p_{i j}=b / n$ otherwise.

Proceeding exactly as in the proof of Theorem 8, we can compare the SDP value for the matrix $\boldsymbol{E}$, to the SDP value for a Gaussian matrix. We obtain the following estimate, whose proof we omit.

Lemma D.2. Let $\boldsymbol{E} \in \mathbb{R}^{n \times n}$ be the random matrix defined above, with $d=(a+b) / 2$, and $\lambda=(a-b) / \sqrt{2(a+b)}$. Let $\boldsymbol{W} \sim \operatorname{GOE}(n)$ be a Gaussian random matrix with $\left(W_{i j}\right)_{i<j} \sim$ $\mathrm{N}(0,1 / n)$. Then, there exists $C=C(\lambda)$ such that, with probability at least $1-C e^{-n / C}$, for all $n \geq n_{0}(a, b)$

$$
\left|\frac{1}{n} \operatorname{SDP}(\boldsymbol{E})-\frac{1}{n} \operatorname{SDP}(\boldsymbol{W})\right| \leq \frac{C \log d}{d^{1 / 10}},
$$

Further $C(\lambda)$ is bounded over compact intervals $\lambda \in\left[0, \lambda_{\max }\right]$.

As a consequence of this lemma, and of Theorem 5, we have

$$
\frac{1}{n} \operatorname{SDP}(\boldsymbol{E}) \leq 2+\frac{C \log d}{d^{1 / 10}},
$$

with probability at least $1-C e^{-n / C}$.

Consider then a maximizer $\boldsymbol{X}_{*}$ of the $\operatorname{SDP}(4)$, with $\boldsymbol{M}=$ $\boldsymbol{A}_{G_{1}}^{\text {cen }}$. We have, by Theorem 5 and Theorem 8 (or, equivalently, by Theorem 3)

$$
\frac{\lambda}{n^{2}}\left\langle\boldsymbol{x}_{0} \boldsymbol{x}_{0}{ }^{\top}, \boldsymbol{X}_{*}\right\rangle+\frac{1}{n}\left\langle\boldsymbol{E}, \boldsymbol{X}_{*}\right\rangle=\frac{1}{n \sqrt{d}} \operatorname{SDP}\left(\boldsymbol{A}^{\text {cen }}\right) \geq 2+\Delta(\varepsilon),
$$

for all $d \geq d_{*}(\varepsilon)$, with probability at least $1-C e^{-n / C}$. Using the bound (124), this implies, for $\lambda$ bounded and some $\Delta_{2}(\varepsilon)>0$

$$
\frac{1}{n^{2}} \sum_{i=1}^{n} \xi_{i}\left\langle\boldsymbol{x}_{0}, \boldsymbol{v}_{i}\right\rangle^{2}=\frac{1}{n^{2}}\left\langle\boldsymbol{x}_{0} \boldsymbol{x}_{0}^{\top}, \boldsymbol{X}_{*}\right\rangle \geq \Delta_{2}(\varepsilon)
$$

Since $\boldsymbol{X}_{*} \in \operatorname{PSD}_{1}(n)$, we have $\xi_{i} \geq 0$ and $\sum_{i=1}^{n} \xi_{i}=n$. Hence there exists $I_{*} \in[n]$ such that

$$
\frac{1}{\sqrt{n}}\left|\left\langle\boldsymbol{x}_{0}, \boldsymbol{v}_{I_{*}}\right\rangle\right| \geq \sqrt{\Delta_{2}(\varepsilon)} .
$$

Assume, without loss of generality, that $\left\langle\boldsymbol{x}_{0}, \boldsymbol{v}_{I_{*}}\right\rangle \geq 0$. Applying Lemma D.1 to the pair $\left(X_{0}, Y\right)$ with joint distribution $n^{-1} \sum_{j=1}^{n} \delta_{x_{0, j}, v_{I_{*}, j}}$, we conclude that there exists $t_{*} \in \mathbb{R}$ such that

$$
\frac{1}{n}\left\langle\boldsymbol{x}_{0}, s_{t_{*}}\left(\boldsymbol{v}_{I_{*}}\right)\right\rangle \geq \frac{\Delta_{2}(\varepsilon)}{4}
$$

(Here $s_{t_{*}}(\cdot)$ is understood to be applied componentwise.) Note that $s_{t_{*}}\left(\boldsymbol{v}_{I_{*}}\right)=\widehat{\boldsymbol{x}}^{\left(I_{*}, J_{*}\right)}$ for some index $J_{*} \in[n]$, whence

$$
\frac{1}{n}\left\langle\boldsymbol{x}_{0}, \widehat{\boldsymbol{x}}^{\left(I_{*}, J_{*}\right)}\right\rangle \geq \frac{\Delta_{2}(\varepsilon)}{4} .
$$

In other words, at least one of the estimators $\left\{\widehat{\boldsymbol{x}}^{(i, j)}: i, j \in\right.$ $[n]\}$ has a good correlation with the ground truth. We are left to prove that step $(i v)$ in our algorithm does indeed select such a pair of indices $i, j$. This follows from the following simple concentration lemma.

Lemma D.3. There exists a constant $C=C(a, b)$ bounded for $a, b$ in bounded intervals, such that, for all $s \in[0,1]$.

$$
\begin{array}{r}
\mathbb{P}\left\{\max _{i, j \in[n]}\left|\left\langle\widehat{\boldsymbol{x}}^{(i, j)}, \boldsymbol{A}_{G_{2}}^{c e n} \widehat{\boldsymbol{x}}^{(i, j)}\right\rangle-\frac{\lambda \sqrt{d}}{2 n}\left\langle\widehat{\boldsymbol{x}}^{(i, j)}, \boldsymbol{x}_{0}\right\rangle^{2}+\frac{\lambda \sqrt{d}}{2}\right|\right. \\
\geq s \sqrt{n}\} \leq C e^{-\sqrt{n} s^{2} / C} .
\end{array}
$$

Proof. Throughout the proof, $C$ denotes a constant that might depend on $a, b$, bounded for $a, b$ in compact intervals. For any fixed vector $\widehat{\boldsymbol{x}} \in\{+1,0,-1\}^{n}$ we have, by AzumaHoeffding inequality

$\mathbb{P}\left\{\left|\left\langle\widehat{\boldsymbol{x}}, \boldsymbol{A}_{G_{2}}^{\mathrm{cen}} \widehat{\boldsymbol{x}}\right\rangle-\mathbb{E}\left\langle\widehat{\boldsymbol{x}}, \boldsymbol{A}_{G_{2}}^{\mathrm{cen}} \widehat{\boldsymbol{x}}\right\rangle\right| \geq s ;\left|E_{2}\right| \leq m_{2}\right\} \leq 2 e^{-\frac{s^{2}}{8 m_{2}}}$

However, by Chernoff bound, $m_{2} \leq C \sqrt{n}$ with probability at least $1-C e^{-n^{1 / 2} / C}$, whence, for all $s \in[0,1]$,

$$
\mathbb{P}\left\{\left|\left\langle\widehat{\boldsymbol{x}}, \boldsymbol{A}_{G_{2}}^{\mathrm{cen}} \widehat{\boldsymbol{x}}\right\rangle-\mathbb{E}\left\langle\widehat{\boldsymbol{x}}, \boldsymbol{A}_{G_{2}}^{\mathrm{cen}} \widehat{\boldsymbol{x}}\right\rangle\right| \geq s \sqrt{n}\right\} \leq C e^{-\sqrt{n} s^{2} / C} .
$$

On the other hand $\mathbb{E} \boldsymbol{A}_{G_{2}}^{\text {cen }}=(a-b)\left(\boldsymbol{x}_{0} \boldsymbol{x}_{0}{ }^{\top}-\mathrm{I}\right) /(2 n)$, whence $\mathbb{P}\left\{\left|\left\langle\widehat{\boldsymbol{x}}, \boldsymbol{A}_{G_{2}}^{\mathrm{cen}} \widehat{\boldsymbol{x}}\right\rangle-\frac{\lambda \sqrt{d}}{2 n}\left\langle\widehat{\boldsymbol{x}}, \boldsymbol{x}_{0}\right\rangle^{2}+\frac{\lambda \sqrt{d}}{2}\right| \geq s \sqrt{n}\right\} \leq C e^{-\frac{\sqrt{n} s^{2}}{C}}$.

The claim follows by taking union bound over $i, j \in[n]$, since $\boldsymbol{x}^{(i, j)}$ is independent of $G_{1}$.

It follows from the last lemma, and Eq. (129) that

$$
\frac{1}{n} \max _{i, j \in[n]}\left\langle\widehat{\boldsymbol{x}}^{(i, j)}, \boldsymbol{A}_{G_{2}}^{\mathrm{cen}} \widehat{\boldsymbol{x}}^{(i, j)}\right\rangle \geq \frac{\lambda \sqrt{d} \Delta_{2}(\varepsilon)^{2}}{64} \equiv \Delta_{3}(\varepsilon),
$$

with probability at least $1-C e^{-n^{1 / 2} / C}$. Hence, again by the last lemma

$$
\begin{aligned}
\max \left\{\left\langle\widehat{\boldsymbol{x}}^{(i, j)}, \boldsymbol{A}_{G_{2}}^{\mathrm{cen}} \widehat{\boldsymbol{x}}^{(i, j)}\right\rangle:(i, j) \in[n], \frac{1}{n}\left|\left\langle\widehat{\boldsymbol{x}}^{(i, j)}, \boldsymbol{x}_{0}\right\rangle\right|\right. & \left.\leq \frac{\Delta_{2}}{8}\right\} \\
& \leq \frac{\Delta_{3}}{2}
\end{aligned}
$$

with probability at least $1-C e^{-n^{1 / 2} / C}$. The claim follows since on the events (133) and (134) we necessarily have $\left|\left\langle\widehat{\boldsymbol{x}}^{(I, J)}, \boldsymbol{x}_{0}\right\rangle\right| \geq n \Delta_{2}(\varepsilon) / 8$. 